\title{
Universal leading quantum correction to the Newton potential
}

\author{
Tibério de Paula Netto ${ }^{1, a}$, Leonardo Modesto ${ }^{1, b}$, Ilya L. Shapiro ${ }^{2,3, c}$ \\ ${ }^{1}$ Department of Physics, Southern University of Science and Technology, Shenzhen 518055, China \\ 2 Present address: Departamento de Física, ICE, Universidade Federal de Juiz de Fora, Juiz de Fora, MG 36036-900, Brazil \\ ${ }^{3}$ Tomsk State Pedagogical University, Tomsk, Russia
}

Received: 29 October 2021 / Accepted: 28 January 2022 / Published online: 21 February 2022

(C) The Author(s) 2022

\begin{abstract}
The derivation of effective quantum gravity corrections to Newton's potential is an important step in the whole effective quantum field theory approach. We hereby add new strong arguments in favor of omitting all the diagrams with internal lines of the massive sources, and we also recalculate the corrections to the Newtonian potential using functional methods in an arbitrary parametrization of the quantum fluctuations of the metric. The general proof of the gauge- and parametrization-independence within this approach is also explicitly given. On top of that, we argue that the universality of the result holds regardless of the details of the ultraviolet completion of quantum gravity theory. Indeed, it turns out that the logarithm quantum correction depends only on the low energy spectrum of the theory that is responsible for the analytic properties of loop's amplitudes.
\end{abstract}

\section{Introduction}

Quantum corrections to Newton's potential for the classical gravitational force is a kind of theoretical "standard candle" for the effective quantum gravity theory. The first calculation of these corrections are half a century old [1], but the explosion of interest to the particular example and the effective quantum gravity in general, started from the seminal works due to Donoghue [2,3]. The total amount of publications (see, e.g., $[4,5]$ and [6] and references therein) is very large, such that we are probably not aware of all of them. However, in our opinion, there remain at least two relevant issues which were not sufficiently well discussed.

First, starting from the paper [7] there was a growing understanding that the gauge-fixing independence of the quantum corrections is an important and useful criterion for the correctness and consistent definition of these corrections.

\footnotetext{
a e-mail: tiberio@sustech.edu.cn

b e-mail: 1modesto@sustech.edu.cn

c e-mail: ilyashapiro2003@ufjf.br (corresponding author)
}

In the mentioned paper, the simplified model of scalar electrodynamics was used to prove that disregarding some of the diagrams is inconsistent as this procedure does not provide the gauge-fixing independence of the potential. In the subsequent papers, i.e., [4,5], all the diagrams were taken into account and the invariance was proved (see, e.g., $[8,9]$ ). However, it turns out that there are serious reasons to take out some of these diagrams $[11,13]$.

The calculations in most of the mentioned (and many other) papers were done using Feynman diagrams where two massive scalar fields model the massive bodies. Then, the complete set of diagrams includes those of $L$ - and $P$ type. The first, $L$-type subset consists of the graphs with internal lines of massless gravitons and massless gauge (Faddeev-Popov) ghosts. These diagrams give only a very small $\mathcal{O}\left(r^{-3}\right)$-type addition to the Newton potential between the two-point masses. Phenomenologically, these graphs may be irrelevant, but conceptually they are the most important ones since the much stronger contributions coming from the $P$-type diagrams should be disregarded because of physical reasons [13]. The point is that our final purpose is to get the quantum corrections to the force between two macroscopic bodies, which are modeled by the scalar field. Taking into account the internal lines of these scalars means that we are regarding the massive macroscopic bodies as quantum objects. For example, if the purpose is to explore the quantum effects on the motion of an asteroid around the Sun, we are quantizing this asteroid and the Sun. Or we are quantizing, e.g., the Earth and the Moon. Indeed, the Moon does not like to be quantized. Thus, we may commit a serious mistake in this way.

Two other arguments for omitting the $P$-type diagrams are as follows. The macroscopic body is made from atoms, and most of the mass of the atom nucleons is due to the quantum effects. Thus, the argument concerning the expansions in $\hbar$ is not operational in this case. Furthermore, nowadays there are strong experimental confirmations of not quantiz- 
ing the classical source in a physically similar situation. It is good to remember the success of the theoretical description of Casimir force (see, e.g., [14]). Despite the existing controversies (see, for example, the critical discussion in [15]), there are no many doubts that the Casimir effect is experimentally detected, and we know that its theoretical description does not involve the quantization of the sources (such as conducting plates) but only the electromagnetic field. There are no reasons to assume that things should be different for the gravitational Casimir effect because the basic formalism is, in general, similar [16]. Thus, the correct procedure is to separate and use only $L$-type diagrams, i.e., those with only massless internal lines. From the quantum field theory viewpoint, this means that the object of our interest is the functional integral

$$
\begin{aligned}
e^{i \Gamma}= & \int d g d \bar{c} d c \exp \left\{i S_{\text {grav }}(g)+i S_{g f}(g)\right. \\
& \left.+i S_{g h}(g, \bar{c}, c)+i S_{\text {sources }}(g, \Phi)\right\},
\end{aligned}
$$

where $g=g_{\alpha \beta}$ is the quantum metric, $\bar{c}=\bar{c}_{\alpha}$ and $c=c^{\beta}$ are gauge ghosts, and $\Phi$ represent the external massive sources. The approach described above was pursued in important work [10] and, most recently, in [11]. Following the last reference, in the calculations described below, we choose these sources as a massive point-like mass and a light point-like test particle, but in principle, the integral (1) can be explored for arbitrary massive sources, e.g., for the dark matter (DM), finite-size stars, or interstellar gas. In all these cases, the integration over $\Phi$ is not included for the reasons described above. This means that the variables $\Phi$ play the role of external parameters, independent of the quantum variables such as the metric components.

A general observation is in order. There are relevant aspects of the semiclassical approach (when metric is a classical background while matter fields quantum), related to the possible transformations which are mixing classical and quantum variables [12]. One can try to elaborate the same idea in the anti-semiclassical approach, when only gravity is quantized. However, in the effective framework, in the IR only the massless fields are relevant, leaving only the effects of graviton and photon [2]. In this effective setting, our antisemiclassical approach emerges naturally and the mixing of the massless degree of freedom with the ones of massive (macroscopic) degrees of freedom describing the classical sources does not look reasonable.

In practice, one can use the background field method for the useful definition and derivation of the integral (1). Then, the effective action in the l.h.s. is $\Gamma(g, \Phi)$ and the integral variables change accordingly. We shall discuss the explicit formulas below.

The paper is organized in the following way. Section 2 describes the derivation of the relevant part of $\Gamma(g, \Phi)$. Sec- tion 3 aims to define the gravitational field in the Newtonian approximation, in the theory with quantum effective contributions. Section 4 describes the motion of test particles and defined the final form of the corrected Newtonian potential. Section 5 is devoted to the general proof of the gauge- and parametrization-independence of the effective quantum correction to Newton's potential. In Section 6 we elaborate on the constrains that an effective field theory should satisfy in order to be consistent in the quantum field theory framework, and after that we explain the universality of the leading correction to Newton's potential, which only depends on the fundamental theory low-energy spectrum. Finally, in Sect. 7 we draw our conclusions.

The notations include the Minkowski metric $\eta_{\mu \nu}=$ $\operatorname{diag}(1,-1,-1,-1)$, while the Riemann curvature tensor is defined as

$$
R_{\cdot \beta \mu \nu}^{\alpha}=\partial_{\mu} \Gamma_{\beta \nu}^{\alpha}-\partial_{\nu} \Gamma_{\beta \mu}^{\alpha}+\Gamma_{\mu \tau}^{\alpha} \Gamma_{\beta \nu}^{\tau}-\Gamma_{\nu \tau}^{\alpha} \Gamma_{\beta \mu}^{\tau} .
$$

The definitions of the Ricci tensor and the scalar curvature are $R_{\mu \nu}=R_{\mu \alpha \nu}^{\alpha}$ and $R=g^{\mu \nu} R_{\mu \nu}$, respectively. We also adopt the units system with $c=1$ and $\hbar=1$.

\section{Derivation of effective action}

In the effective quantum gravity $[3,6]$, it is assumed that the low-energy effects are described by Einstein's general relativity with zero cosmological constant because this term is irrelevant at the astrophysical scale where the Newtonian limit applies. The possible contributions of higher derivative terms either decouple (see, e.g., [17] for preliminary analysis, coherent with the standard approach in particle physics [18, 19]) or regarded as small perturbations by definition [20]. The reader can consult [21] and [22] for a general discussion of different approaches. However, the net result is that we have to start from the classical action of the form

$S=-\frac{1}{\kappa^{2}} \int d^{4} x \sqrt{-g} R+S_{\text {sources }}(g, \Phi)$,

where $\kappa^{2}=16 \pi G$ and $G$ is the Newton constant. As the source term, we shall consider the action of the free massive particle $S_{M}$, with coordinates $y^{\mu}$

$S_{M}=-M \int d s=-M \int \sqrt{g_{\mu \nu} d y^{\mu} d y^{\nu}}$.

To quantize the gravitational field, we apply the background field method with the most general (at the one-loop level) parametrization of the metric, as formulated in Ref. [23] (see also [24]), namely

$$
\begin{aligned}
& g_{\mu \nu} \longrightarrow g_{\mu \nu}^{\prime}=e^{2 \gamma_{0} \kappa \sigma}\left[g_{\mu \nu}+\kappa\left(\gamma_{1} \phi_{\mu \nu}+\gamma_{2} \phi g_{\mu \nu}\right)\right. \\
& \left.\quad+\kappa^{2}\left(\gamma_{3} \phi_{\mu \rho} \phi_{\nu}^{\rho}+\gamma_{4} g_{\mu \nu} \phi_{\rho \sigma} \phi^{\rho \sigma}+\gamma_{5} \phi \phi_{\mu \nu}+\gamma_{6} g_{\mu \nu} \phi^{2}\right)\right] .
\end{aligned}
$$


In this formula, $g_{\mu \nu}$ is the background field and $\sigma, \phi_{\mu \nu}$ are quantum fields. The indices are lowered and raised with the background metric $g_{\mu \nu}$. Also, $\phi=g_{\mu \nu} \phi^{\mu \nu}$. Finally, $\gamma_{0,1, \ldots, 6}$ are arbitrary coefficients which parameterize quantum variables.

Treating the conformal factor $\sigma$ as a new field introduces an artificial conformal symmetry [25]. In order to remove the corresponding degeneracy, we implement the conformal gauge fixing

$\sigma=\lambda \phi$,

where $\lambda$ is a gauge-fixing parameter. Using (6) and expanding the exponential, one can rewrite Eq. (5) in the form

$g_{\mu \nu}^{\prime}=g_{\mu \nu}+\kappa A_{\mu \nu}^{\alpha \beta} \phi_{\alpha \beta}+\kappa^{2} \phi_{\lambda \tau} B_{\mu \nu}^{\lambda \tau, \rho \sigma} \phi_{\rho \sigma}+\mathcal{O}\left(\kappa^{3}\right)$,

where

$A_{\mu \nu}^{\alpha \beta}=\gamma_{1} \delta_{\mu \nu}^{\alpha \beta}+\left(\gamma_{2}+2 \gamma_{0} \lambda\right) g_{\mu \nu} g^{\alpha \beta}$,

and

$$
\begin{aligned}
B_{\lambda \tau}^{\mu \nu, \alpha \beta}= & \frac{\gamma_{3}}{4}\left(\delta_{\lambda \tau}^{\mu \alpha} g^{\nu \beta}+\delta_{\lambda \tau}^{\nu \alpha} g^{\mu \beta}+\delta_{\lambda \tau}^{\mu \beta} g^{\nu \alpha}+\delta_{\lambda \tau}^{\nu \beta} g^{\mu \alpha}\right) \\
& +\gamma_{4} \delta^{\mu \nu, \alpha \beta} g_{\lambda \tau}+\frac{1}{2}\left(\gamma_{5}+2 \gamma_{0} \lambda \gamma_{1}\right) \\
& \left(\delta^{\mu \nu}{ }_{\lambda \tau} g^{\alpha \beta}+\delta_{\lambda \tau}^{\alpha \beta} g^{\mu \nu}\right) \\
& +\left(\gamma_{6}+2 \gamma_{0} \lambda \gamma_{2}+2 \gamma_{0}^{2} \lambda^{2}\right) g^{\mu \nu} g^{\alpha \beta} g_{\lambda \tau}
\end{aligned}
$$

with

$\delta_{\alpha \beta}^{\mu \nu}=\frac{1}{2}\left(\delta_{\alpha}^{\mu} \delta_{\beta}^{\nu}+\delta_{\beta}^{\mu} \delta_{\alpha}^{\nu}\right)$.

To fix the diffeomorphism invariance and simplify the calculations, ${ }^{1}$ we introduce the following minimal gauge fixing action [23],

$$
\begin{aligned}
S_{g f}= & -\frac{1}{2} \int d^{4} x \sqrt{-g}\left[\gamma_{1} \nabla_{\lambda} \phi_{\mu}^{\lambda}\right. \\
& \left.-\frac{1}{2}\left(\gamma_{1}+2 \gamma_{2}+4 \gamma_{0} \lambda\right) \nabla_{\mu} \phi\right]^{2} .
\end{aligned}
$$

Performing the expansion of (5) in (3) with the minimal gauge-fixing term, we arrive at the bilinear, in the quantum metric, part of the action

$\left(S+S_{g f}\right)^{(2)}=\frac{1}{2} \int d^{4} x \sqrt{-g} \phi_{\mu \nu} \mathbf{H}^{\mu v, \alpha \beta} \phi_{\alpha \beta}$,

where

$$
\mathbf{H}^{\mu v, \alpha \beta}=-\left(\mathbf{K}^{\mu v, \alpha \beta} \square+\Pi^{\mu v, \alpha \beta}+\mathbf{M}^{\mu v, \alpha \beta}\right),
$$

with

$$
\mathbf{K}^{\mu \nu, \alpha \beta}=A_{\lambda \tau}^{\mu \nu} K^{\lambda \tau, \rho \sigma} A_{\rho \sigma}^{\alpha \beta},
$$

1 The non-minimal gauge was considered in [11] and proved to give a gauge-independent result for the one-loop contribution to Newton's gravitational potential, so we avoid discussing it here.

$$
\begin{aligned}
& \Pi^{\mu \nu, \alpha \beta}=A_{\lambda \tau}^{\mu \nu} K^{\lambda \tau, \rho \sigma} A_{\rho \sigma}^{\alpha \beta}-2 B_{\lambda \tau}^{\mu \nu, \alpha \beta} G^{\mu \nu}, \\
& \mathbf{M}^{\mu \nu, \alpha \beta}=\kappa^{2}\left(B_{\lambda \tau}^{\mu \nu, \alpha \beta} T^{\lambda \tau}-A_{\lambda \tau}^{\mu \nu} M^{\lambda \tau, \rho \sigma} A_{\rho \sigma}^{\alpha \beta}\right),
\end{aligned}
$$

where we used the notations:

$$
\begin{aligned}
G^{\mu \nu}= & R^{\mu \nu}-\frac{1}{2} g^{\mu \nu} R, \\
& K^{\mu \nu, \alpha \beta}=\frac{1}{2}\left(\delta^{\mu \nu, \alpha \beta}-\frac{1}{2} g^{\mu \nu} g^{\alpha \beta}\right), \\
\Pi^{\mu \nu, \alpha \beta}= & \frac{1}{2}\left(R^{\mu \alpha \nu \beta}+R^{\mu \beta \nu \alpha}\right) \\
& +\frac{1}{4}\left(g^{\mu \alpha} R^{\nu \beta}+g^{\mu \beta} R^{\nu \alpha}+g^{\nu \alpha} R^{\mu \beta}+g^{\nu \beta} R^{\mu \alpha}\right) \\
& -\frac{1}{2}\left(g^{\mu \nu} R^{\alpha \beta}+g^{\alpha \beta} R^{\mu \nu}\right)-K^{\mu \nu, \alpha \beta} R .
\end{aligned}
$$

Furthermore, in the source sector,

$T^{\mu \nu}(x)=M \int d s \delta(x-y(s)) u^{\mu} u^{\nu}$

is the point-like mass energy-momentum tensor and

$M^{\mu v, \alpha \beta}(x)=\frac{M}{4} \int d s \delta(x-y(s)) u^{\mu} u^{v} u^{\alpha} u^{\beta}$.

It is worth noting that the boldface notation are used for the operators depending on the parametrization and gauge fixing. Also, in (16) and (17),

$u^{\mu}=\frac{d y^{\mu}}{d s}$

is the particle four-velocity and the delta function is introduced through the relation

$\int d^{4} x \sqrt{-g(x)} \delta(x-y(s))=1$.

The standard Schwinger-DeWitt technique for the oneloop divergences requires to reduce the Hessian (13) to the standard expression for the minimal operator, i.e., $\hat{1} \square+$ $\hat{\mathbf{P}}-(1 / 6) \hat{1} R$. Therefore, we multiply (13) with the negative inverse of (14), namely

$\mathbf{K}_{\mu \nu, \alpha \beta}^{-1}=\left(A^{-1}\right)_{\mu \nu}^{\lambda \tau} K_{\lambda \tau, \rho \sigma}^{-1}\left(A^{-1}\right)_{\alpha \beta}^{\rho \sigma}$,

where

$$
\begin{aligned}
& K_{\lambda \tau, \rho \sigma}^{-1}=2\left(\delta_{\lambda \tau, \rho \sigma}-\frac{1}{2} g_{\lambda \tau} g_{\rho \sigma}\right) \\
& \left(A^{-1}\right)_{\mu \nu}^{\alpha \beta}=\frac{1}{\gamma_{1}}\left(\delta^{\alpha \beta}{ }_{\mu \nu}-\frac{\gamma_{2}+2 \gamma_{0} \lambda}{\gamma_{1}+4 \gamma_{2}+8 \gamma_{0} \lambda} g_{\mu \nu} g^{\alpha \beta}\right) .
\end{aligned}
$$

Adopting the suppressed index notation of Ref. [26], we get $\hat{\mathbf{H}} \equiv-\mathbf{K}_{\mu \nu, \lambda \tau}^{-1} \cdot \mathbf{H}^{\lambda \tau, \alpha \beta}=\hat{1} \square+\hat{\mathbf{P}}-\frac{1}{6} \hat{1} R$, 
where $\hat{1}=\delta^{\alpha \beta}{ }_{\mu \nu}$ is the identity matrix for symmetric rank-2 tensors and

$\hat{\mathbf{P}}=\mathbf{K}_{\mu \nu, \lambda \tau}^{-1} \cdot \boldsymbol{\Pi}^{\lambda \tau, \alpha \beta}+\mathbf{K}_{\mu \nu, \lambda \tau}^{-1} \cdot \mathbf{M}^{\lambda \tau, \alpha \beta}+\frac{1}{6} \hat{1} R$.

Performing the substitution (5) in (1), we have the oneloop contribution to the effective action

$\bar{\Gamma}^{(1)}=\frac{i}{2} \operatorname{Tr} \ln \hat{\mathbf{H}}-i \operatorname{Tr} \ln \hat{\mathbf{H}}_{g h}$,

where $\hat{\mathbf{H}}_{g h}$ is the bilinear part of the Faddeev-Popov ghost action. The contribution of this term to (24) is the same as in Ref. [23] hence we do not repeat it here.

The divergent part of the first term in (24) can be evaluated by the trace of the coincidence limit of the Schwinger-DeWitt expansion $\hat{a}_{2}$ coefficient [27] (we do not include here the surface terms, as they are irrelevant for our purpose)

$$
\begin{aligned}
& \left.\frac{i}{2} \operatorname{Tr} \ln \hat{\mathbf{H}}\right|_{d i v}=-\frac{\mu^{n-4}}{(4 \pi)^{2}(n-4)} \int d^{n} x \sqrt{-g} \operatorname{tr} \\
& \quad \times\left\{\frac{1}{180} \hat{1}\left(R_{\mu \nu \alpha \beta}^{2}-R_{\mu \nu}^{2}\right)+\frac{1}{2} \hat{\mathbf{P}}^{2}+\frac{1}{12} \hat{\mathcal{R}}_{\mu \nu}^{2}\right\},
\end{aligned}
$$

where

$\hat{\mathcal{R}}_{\lambda \tau}=-\frac{1}{2}\left(\delta_{\mu}^{\alpha} R_{\cdot \nu \rho \sigma}^{\beta}+\delta_{\mu}^{\beta} R_{\cdot \nu \rho \sigma}^{\alpha}+\delta_{\nu}^{\alpha} R_{\cdot \mu \rho \sigma}^{\beta}+\delta_{\nu}^{\beta} R_{\cdot \mu \rho \sigma}^{\alpha}\right)$

and $\mu$ is the renormalization group scale.

To simplify (25), we use the identity $g_{\mu \nu} u^{\mu} u^{\nu}=1$ which implies in the following equations for the expressions (16) and (17):

$$
\begin{aligned}
g_{\mu \nu} M^{\mu \nu, \alpha \beta} & =\frac{1}{4} T^{\alpha \beta}, \\
M_{\mu \nu, \alpha \beta} M^{\mu \nu, \alpha \beta} & =\frac{1}{16} T_{\mu \nu} T^{\mu \nu}, \\
T_{\mu \nu} T^{\mu \nu} & =T^{2},
\end{aligned}
$$

where

$T \equiv T_{\mu}^{\mu}=M \int d s \delta(x-y(s))$

is the trace of the energy-momentum tensor for a massive point-like particle. There are also equations analogous to (27) for other index contractions since $M^{\mu \nu, \alpha \beta}$ is totally symmetric.

The evaluation of (25) follows the scheme explained in detail in [23]. Thus, skipping the algebra, we get, for the one-loop divergences

$$
\begin{aligned}
\bar{\Gamma}_{d i v}^{(1)}= & -\frac{\mu^{n-4}}{(4 \pi)^{2}(n-4)} \int d^{n} x \sqrt{-g} \\
& \times\left\{c_{1} R_{\mu \nu \alpha \beta}^{2}+c_{2} R_{\mu \nu}^{2}+c_{3} R^{2}\right. \\
& \left.+\kappa^{2} c_{4} R_{\mu \nu} T^{\mu \nu}+\kappa^{2} c_{5} R T+\kappa^{4} c_{6} T^{2}\right\},
\end{aligned}
$$

where the dimensionless coefficients are given by

$c_{1}=\frac{53}{45}, \quad c_{2}=-\frac{361}{90}-\frac{4 \xi_{2}}{\gamma_{1}^{4} Z^{2}}, \quad c_{3}=\frac{43}{36}+\frac{\xi_{3}}{3 \gamma_{1}^{4} Z^{4}}$,

$c_{4}=-\frac{2 \xi_{4}}{\gamma_{1}^{4} Z^{4}}, \quad c_{5}=\frac{5}{24}-\frac{\xi_{5}}{6 \gamma_{1}^{4} Z^{4}}, \quad c_{6}=\frac{1}{32}-\frac{\xi_{6}}{4 \gamma_{1}^{4} Z^{4}}$,

where $Z=\gamma_{1}+4 \gamma_{2}+8 \gamma_{0} \lambda$, and the bulky expressions for the parametrization dependent coefficients $\xi_{i}$ can be found in Appendix A.

In the limit $\gamma_{1} \rightarrow 1, \gamma_{2}, \ldots, 6 \rightarrow 0$ and $\gamma_{0} \rightarrow 0$ for the parameters of (5), Eq. (31) reproduces the result of Ref. [11], evaluated in the standard parametrization in the minimal gauge.

Using the classical equations of motion

$$
R_{\mu \nu}-\frac{1}{2} g_{\mu \nu} R=\frac{\kappa^{2}}{2} T_{\mu \nu}
$$

and (29), Eq. (24) boils down to

$$
\begin{aligned}
\left.\bar{\Gamma}_{\text {div }}^{(1)}\right|_{\text {on-shell }}= & -\frac{\mu^{n-4}}{(4 \pi)^{2}(n-4)} \int d^{n} x \sqrt{-g} \\
& \times\left\{\frac{53}{45} R_{\mu \nu \alpha \beta}^{2}-\frac{373}{480} \kappa^{4} T^{2}\right\},
\end{aligned}
$$

independently of the choice of parametrization and gauge parameters, exactly as it should be.

To consider the Newtonian limit it is useful to rewrite Eq. (31) in a more convenient Weyl basis, using the relations

$$
R_{\mu \nu \alpha \beta}^{2}=2 C^{2}-E+\frac{1}{3} R^{2}, \quad R_{\mu \nu}^{2}=\frac{1}{2} C^{2}-\frac{1}{2} E+\frac{1}{3} R^{2} .
$$

Here $C^{2}=R_{\mu \nu \alpha \beta}^{2}-2 R_{\mu \nu}^{2}+1 / 3 R^{2}$ is the square of the Weyl tensor and $E=R_{\mu \nu \alpha \beta}^{2}-4 R_{\mu \nu}^{2}+R^{2}$ is the integrand of the (topological at $n=4$ ) Gauss-Bonnet term. Discarding the non essential for us topological term, we get for (31):

$$
\begin{aligned}
\bar{\Gamma}_{d i v}^{(1)}= & -\frac{\mu^{n-4}}{n-4} \int d^{n} x \sqrt{-g}\left\{\beta_{2} C^{2}-\frac{1}{3} \beta_{0} R^{2}\right. \\
& \left.-2 \kappa^{2} \beta_{R T 1} R_{\mu \nu} T^{\mu \nu}+\kappa^{2} \beta_{R T 2} R T+\kappa^{4} \beta_{T T} T^{2}\right\},
\end{aligned}
$$

with the following relations between the coefficients,

$$
\begin{aligned}
\beta_{2} & =\frac{4 c_{1}+c_{2}}{2(4 \pi)^{2}}, \quad \beta_{0}=-\frac{c_{1}+c_{2}+3 c_{3}}{(4 \pi)^{2}}, \\
\beta_{R T 1} & =-\frac{c_{4}}{2(4 \pi)^{2}}, \quad \beta_{R T 2}=\frac{c_{5}}{(4 \pi)^{2}}, \quad \beta_{T T}=\frac{c_{6}}{(4 \pi)^{2}} .
\end{aligned}
$$

To reconstruct the effective action we use the rule (see, e.g., [22] for the introduction and further references, including the generalizations for the loops of massive fields)

$\frac{\mu^{n-4}}{n-4} \longrightarrow \frac{1}{2} \ln \left(\frac{\square}{\mu^{2}}\right)$ 
that is valid for the massless fields contributions such as the graviton. In this way, we finally get

$$
\begin{aligned}
\bar{\Gamma}^{(1)}= & -\int d^{4} x \sqrt{-g}\left\{\frac{1}{2} \beta_{2} C_{\mu \nu \alpha \beta} \ln \left(\frac{\square}{\mu^{2}}\right) C^{\mu \nu \alpha \beta}\right. \\
& -\frac{1}{6} \beta_{0} R \ln \left(\frac{\square}{\mu^{2}}\right) R \\
& -\kappa^{2} \beta_{R T 1} R_{\mu \nu} \ln \left(\frac{\square}{\mu^{2}}\right) T^{\mu \nu}+\frac{1}{2} \kappa^{2} \beta_{R T 2} R \ln \left(\frac{\square}{\mu^{2}}\right) T \\
& \left.+\frac{1}{2} \kappa^{4} \beta_{T T} T \ln \left(\frac{\square}{\mu^{2}}\right) T\right\} .
\end{aligned}
$$

\section{Gravitational field in the Newtonian limit with quantum contributions}

Here we are going to obtain the gravitational field in the Newtonian limit taking into account one-loop quantum corrections. The effective action is given by the classical action $S$, defined in (3), and the one-loop correction (40), i.e.,

$\Gamma=S+\bar{\Gamma}^{(1)}$.

To obtain the metric potentials with logarithm quantum corrections, we follow Refs. [29,30] (see also [31-33]). First, we consider small perturbations of the background metric around the Minkowiski spacetime,

$g_{\mu \nu}=\eta_{\mu \nu}+\kappa h_{\mu \nu}, \quad\left|\kappa h_{\mu \nu}\right| \ll 1$

and expand the action (41) to the relevant order in the weakfield approximation. In this way, we get

$$
\begin{aligned}
\Gamma= & -\frac{1}{2} \int d^{4} x\left\{\frac{1}{2} h_{\mu \nu} f_{2}(\square) \square h^{\mu \nu}-h^{\mu v} f_{2}(\square) \partial_{\mu} \partial_{\lambda} h_{v}^{\lambda}\right. \\
& -\frac{1}{6} h\left[f_{2}(\square)+2 f_{0}(\square)\right] \square h \\
& +\frac{1}{3} h\left[f_{2}(\square)+2 f_{0}(\square)\right] \partial_{\mu} \partial_{\nu} h^{\mu \nu} \\
& \left.+\frac{1}{3} h_{\alpha \beta}\left[f_{2}(\square)-f_{0}(\square)\right] \frac{\partial^{\alpha} \partial^{\beta} \partial^{\mu} \partial^{\nu}}{\square} h_{\mu \nu}\right\} \\
& -\frac{\kappa}{2} \int d^{4} x\left\{h_{\mu \nu} f_{R T 1}(\square) T^{\mu \nu}+\kappa^{2} \beta_{R T 2} h_{\mu \nu}\right. \\
& \left.\ln \left(\frac{\square}{\mu^{2}}\right)\left(\partial^{\mu} \partial^{\nu}-\eta^{\mu v} \square\right) T\right\},
\end{aligned}
$$

where we defined the form factors

$f_{i}(\square)=1+\kappa^{2} \beta_{i} \ln \left(\frac{\square}{\mu^{2}}\right) \square, \quad i=2,0, R T 1$.

Let us note that in (43), $\square=\eta^{\mu v} \partial_{\mu} \partial_{\nu}$ is the flat space d'Alembertian. Also, the term proportional to $\beta_{T T}$ is discarded here because it is $\propto M^{2}$ and, therefore, is beyond the weak-field approximation.

Then, taking the functional derivative with respect to $h_{\mu \nu}$ we get the equations of motion

$$
\begin{aligned}
f_{2}(\square)\left(\square h^{\mu \nu}-\partial^{\mu} \partial^{\lambda} h_{\lambda}^{\nu}-\partial^{v} \partial^{\lambda} h_{\lambda}^{\mu}\right) \\
+\frac{1}{3}\left[f_{2}(\square)+2 f_{0}(\square)\right]\left[\eta^{\mu v}\left(\partial^{\alpha} \partial^{\beta} h_{\alpha \beta}-\square h\right)+\partial^{\mu} \partial^{v} h\right] \\
+\frac{2}{3}\left[f_{2}(\square)-f_{0}(\square)\right] \frac{\partial^{\mu} \partial^{v} \partial^{\alpha} \partial^{\beta}}{\square} h_{\alpha \beta} \\
=-\kappa\left[f_{R T 1}(\square) T^{\mu v}+\kappa^{2} \beta_{R T 2} \ln \left(\frac{\square}{\mu^{2}}\right)\left(\partial^{\mu} \partial^{v}-\eta^{\mu v} \square\right) T\right] .
\end{aligned}
$$

The second step is to consider the perturbation in the isotropic Newtonian form

$\kappa h_{00}=2 \varphi(r), \quad \kappa h_{i j}=2 \delta_{i j} \psi(r)$

and choose, as a source of the gravitational potentials, a static point-like mass located at the origin. In this case, $u^{\mu}=\delta_{0}^{\mu}$, and Eq. (16) becomes

$T^{\mu \nu}=\delta_{0}^{\mu} \delta_{0}^{\nu} \rho, \quad \rho=M \delta(\mathbf{r})$.

The metric potentials (46) can be obtained from the 00 component and the trace of Eq. (45), which are given respectively by

$$
\begin{aligned}
& {\left[f_{2}(-\Delta)-f_{0}(-\Delta)\right] \Delta \varphi+\left[f_{2}(-\Delta)+2 f_{0}(-\Delta)\right] \Delta \psi=} \\
& =\frac{3 \kappa^{2}}{4}\left[f_{R T 1}(-\Delta)+\kappa^{2} \beta_{R T 2} \ln \left(-\frac{\Delta}{\mu^{2}}\right) \Delta\right] \rho \\
& f_{0}(-\Delta)(\Delta \varphi-2 \Delta \psi) \\
& =-\frac{\kappa^{2}}{4}\left[f_{R T 1}(-\Delta)+3 \kappa^{2} \beta_{R T 2} \ln \left(-\Delta / \mu^{2}\right)\right] \rho
\end{aligned}
$$

where we traded $\square$ for $-\Delta$ since the metric is static.

To solve (48) and (49) we perform the loop expansion of the potentials,

$$
\begin{aligned}
\varphi & =\varphi^{(0)}+\varphi^{(1)}+\mathcal{O}\left(\hbar^{2}\right), \\
\psi & =\psi^{(0)}+\psi^{(1)}+\mathcal{O}\left(\hbar^{2}\right),
\end{aligned}
$$

where $\varphi^{(l)}$ and $\psi^{(l)}$ are of the order $\mathcal{O}\left(\hbar^{l}\right)$. Since $\beta_{i}=\mathcal{O}(\hbar)$, we get the equations at zero and first orders in $\hbar$ in the form

$$
\begin{aligned}
& \Delta \varphi^{(0)}=\Delta \psi^{(0)}=\frac{\kappa^{2} M}{4} \delta(\mathbf{r}) \\
& \Delta \psi^{(1)}=\frac{\kappa^{2} \beta_{2}}{3} \ln \left(-\frac{\Delta}{\mu^{2}}\right) \Delta^{2}\left(\varphi^{(0)}+\psi^{(0)}\right) \\
& -\frac{\kappa^{2} \beta_{0}}{3} \ln \left(-\frac{\Delta}{\mu^{2}}\right) \Delta^{2}\left(\varphi^{(0)}-2 \psi^{(0)}\right) \\
& +\frac{\kappa^{4} M}{4}\left(-\beta_{R T 1}+\beta_{R T 2}\right) \ln \left(-\frac{\Delta}{\mu^{2}}\right) \Delta \delta(\mathbf{r}) \\
& \Delta \varphi^{(1)}-2 \Delta \psi^{(1)}=\kappa^{2} \beta_{0} \ln \left(-\frac{\Delta}{\mu^{2}}\right) \Delta^{2}\left(\varphi^{(0)}-2 \psi^{(0)}\right) \\
& +\frac{\kappa^{4} M}{4}\left(\beta_{R T 1}-3 \beta_{R T 2}\right) \ln \left(-\frac{\Delta}{\mu^{2}}\right) \Delta \delta(\mathbf{r})
\end{aligned}
$$


In the three-dimensional Fourier space, we obtain the following solutions for the transformed potentials:

$$
\begin{aligned}
& \varphi^{(0)}(k)=\psi^{(0)}(k)=-\frac{\kappa^{2} M}{4 k^{2}}, \\
& \varphi^{(1)}(k)=\frac{\kappa^{4} M}{4}\left(\frac{4}{3} \beta_{2}-\frac{1}{3} \beta_{0}-\beta_{R T 1}-\beta_{R T 2}\right) \ln \left(\frac{k^{2}}{\mu^{2}}\right), \\
& \psi^{(1)}(k)=\frac{\kappa^{4} M}{4}\left(\frac{2}{3} \beta_{2}+\frac{1}{3} \beta_{0}-\beta_{R T 1}+\beta_{R T 2}\right) \ln \left(\frac{k^{2}}{\mu^{2}}\right),
\end{aligned}
$$

where $k=|\mathbf{k}|$. Next, using the following inverse Fourier transforms,

$$
\begin{aligned}
& \int \frac{d^{3} k}{(2 \pi)^{3}} e^{-i \mathbf{k} \cdot \mathbf{r}} \frac{1}{k^{2}}=\frac{1}{4 \pi r}, \\
& \int \frac{d^{3} k}{(2 \pi)^{3}} e^{-i \mathbf{k} \cdot \mathbf{r}} \ln \left(\frac{k^{2}}{\mu^{2}}\right)=-\frac{1}{2 \pi r^{3}} \quad(r \neq 0),
\end{aligned}
$$

we arrive at the general results

$$
\begin{aligned}
& \varphi(r)=-\frac{\kappa^{2} M}{16 \pi r}-\left(\frac{4}{3} \beta_{2}-\frac{1}{3} \beta_{0}-\beta_{R T 1}-\beta_{R T 2}\right) \frac{\kappa^{4} M}{8 \pi r^{3}}, \\
& \psi(r)=-\frac{\kappa^{2} M}{16 \pi r}-\left(\frac{2}{3} \beta_{2}+\frac{1}{3} \beta_{0}-\beta_{R T 1}+\beta_{R T 2}\right) \frac{\kappa^{4} M}{8 \pi r^{3}} .
\end{aligned}
$$

We do not write the explicit expression for the potentials in terms of the parameters $\gamma_{1, \ldots, 6}, \gamma_{0}$ and $\lambda$ since they are too cumbersome and not very illuminating. At the same time, it is easy to see, using Eqs. (37)-(38), that the obtained potentials depend on the parametrization and gauge-fixing parameters. On the other hand, the potentials $\varphi(r)$ and $\psi(r)$ do not depend on the parametrization of the background field (42). Indeed, the dependence on the parametrization of the quantum fields and the gauge-fixing should be expected, because the effective equations of motion are gauge and parametrization dependent [28].

In the standard $g_{\mu \nu}+\phi_{\mu \nu}$ parametrization limit, $\gamma_{1} \rightarrow 1$, $\gamma_{2, \ldots, 6} \rightarrow 0$ and $\gamma_{0} \rightarrow 0$. Then, Eqs. (57) and (58) become

$$
\begin{aligned}
& \varphi(r)=-\frac{G M}{r}\left(1+\frac{61}{60} \frac{G}{\pi r^{2}}\right), \\
& \psi(r)=-\frac{G M}{r}\left(1+\frac{23}{60} \frac{G}{\pi r^{2}}\right) .
\end{aligned}
$$

Formula (59) matches the result presented in [11] for the $h_{00}$ component of the background metric evaluated in the standard parametrization with the minimal DeWitt gauge.

\section{The motion of a test particle}

Let us now follow the main idea of Ref. [11] that even though the loop corrections to the gravitational field generated by a point-like mass is parametrization and gauge-fixing dependent, the physical observables should be invariant. As an example, consider the acceleration of a test particle moving in the gravitational field described in the previous section. The key observation is that the test particle also couples with the quantum metric and, as a consequence of this, its geodesic equation receives quantum corrections. Thus, the two types of quantum corrections are supposed to combine into the invariant result. Finally, in the non-relativistic limit, we expect to meet invariant quantum corrections to Newton's law, $m \mathbf{a}=\mathbf{F}$.

The classical action for a test particle is given by equation (4), but with another mass,

$S_{m}=-m \int \sqrt{g_{\mu \nu} d x^{\mu} d x^{\nu}}$,

and coordinates $x^{\mu}=(t, \mathbf{r})$. We assume that $m \ll M$, such that we can neglect contribution of the small mass $m$ to the potentials (57) and (58). The one-loop corrections to (61) can be obtained from (40) by the substitution $T^{\mu \nu} \longrightarrow$ $T^{\mu \nu}+T_{m}^{\mu \nu}[11]$, where

$T_{m}^{\mu \nu}=m \int d s \delta(y-x(s)) u^{\mu} u^{\nu}$.

Applying this procedure yields

$$
\begin{aligned}
\bar{\Gamma}_{m}^{(1)}= & \int d^{4} x \sqrt{-g}\left\{\kappa^{2} \beta_{R T 1} R_{\mu \nu} \ln \left(\frac{\square}{\mu^{2}}\right) T_{m}^{\mu \nu}\right. \\
& \left.-\frac{1}{2} \kappa^{2} \beta_{R T 2} R \ln \left(\frac{\square}{\mu^{2}}\right) T_{m}-\kappa^{4} \beta_{T T} T \ln \left(\frac{\square}{\mu^{2}}\right) T_{m}\right\} .
\end{aligned}
$$

The total action for the test particle is $\Gamma_{m}=S_{m}+\bar{\Gamma}_{m}^{(1)}$. Taking the functional derivative with respect to $x_{\mu}$, we find

$$
\frac{1}{m} \frac{\delta \Gamma_{m}}{\delta x_{\mu}}=-\left(\frac{d^{2} x^{\mu}}{d s^{2}}+\Gamma_{\alpha \beta}^{\mu} \frac{d x^{\alpha}}{d s} \frac{d x^{\beta}}{d s}\right)+\frac{1}{m} \frac{\delta \bar{\Gamma}_{m}^{(1)}}{\delta x_{\mu}}=0,
$$

where $\Gamma_{\alpha \beta}^{\mu}$ is the Christoffel symbol. In the non-relativistic limit, this equation boils down to

$\mathbf{a}-\frac{1}{m} \frac{\delta \bar{\Gamma}_{m}^{(1)}}{\delta \mathbf{r}}=-\nabla \varphi, \quad$ where $\quad \mathbf{a}=\frac{d^{2} \mathbf{r}}{d t^{2}}$

is the particle acceleration.

To evaluate the functional derivative in (65), all the metric functions in Eq. (63) should be taken at the order $\mathcal{O}\left(\hbar^{0}\right)$, owing to the one-loop approximation and the fact $\beta_{i} \sim \mathcal{O}(\hbar)$. On top of that, in the non-relativistic limit we can take $s=t$, $u^{\mu}=(1,0)$. Thus, using Eq. (47) for the source energymomentum tensor, we get for (63), in the weak-field approximation,

$$
\begin{aligned}
\bar{\Gamma}_{m}^{(1)}= & m \int d t\left\{\kappa^{2} \beta_{R T 1} \ln \left(\frac{-\Delta}{\mu^{2}}\right) R_{00}^{(0)}(\mathbf{r}(t))\right. \\
& -\frac{1}{2} \kappa^{2} \beta_{R T 2} \ln \left(\frac{-\Delta}{\mu^{2}}\right) R^{(0)}(\mathbf{r}(t)) \\
& \left.-\kappa^{4} \beta_{T T} M \ln \left(\frac{-\Delta}{\mu^{2}}\right) \delta(\mathbf{r}(t))\right\} .
\end{aligned}
$$


The $\mathcal{O}\left(\hbar^{0}\right)$ curvatures can be evaluated through the relations for the 00-component of the Ricci tensor and for the scalar curvature and also using Eq. (52),

$R_{00}^{(0)}(\mathbf{r}(t))=\Delta \varphi^{(0)}=\frac{\kappa^{2} M}{4} \delta(\mathbf{r}(t))$,

$R^{(0)}(\mathbf{r}(t))=2\left(\Delta \varphi^{(0)}-2 \Delta \psi^{(0)}\right)=-\frac{\kappa^{2} M}{2} \delta(\mathbf{r}(t))$.

Finally, using the Eq. (56) in the coordinate space,

$\ln \left(-\frac{\Delta}{\mu^{2}}\right) \delta(\mathbf{r})=-\frac{1}{2 \pi r^{3}}$,

we find

$\bar{\Gamma}_{m}^{(1)}=-\frac{\kappa^{4} m M}{8 \pi}\left(-\beta_{R T 1}-\beta_{R T 2}+4 \beta_{T T}\right) \int \frac{d t}{r^{3}(t)}$,

implying that

$\frac{1}{m} \frac{\delta \bar{\Gamma}_{m}^{(1)}}{\delta \mathbf{r}}=-\frac{\kappa^{4} M}{8 \pi}\left(-\beta_{R T 1}-\beta_{R T 2}+4 \beta_{T T}\right) \nabla\left(\frac{1}{r^{3}}\right)$.

Plugging this equation into (65) and using Eq. (57), we get

$\mathbf{a}=-\nabla\left[-\frac{\kappa^{2} M}{16 \pi r}-\left(\frac{4}{3} \beta_{2}-\frac{1}{3} \beta_{0}-2 \beta_{R T 1}-2 \beta_{R T 2}+4 \beta_{T T}\right) \frac{\kappa^{4} M}{8 \pi r^{3}}\right]$.

The quantum corrected Newtonian potential is defined through the relation [11]

$\mathbf{a} \equiv-\nabla V$.

Thus, (71) and (72) give

$$
\begin{aligned}
V(r)= & -\frac{\kappa^{2} M}{16 \pi r}-\left(\frac{4}{3} \beta_{2}-\frac{1}{3} \beta_{0}-2 \beta_{R T 1}-2 \beta_{R T 2}+4 \beta_{T T}\right) \\
& \times \frac{\kappa^{4} M}{8 \pi r^{3}} .
\end{aligned}
$$

Finally, using Eqs. (37)-(38) we get

$V(r)=-\frac{G M}{r}\left(1+\frac{17}{20} \frac{G}{\pi r^{2}}\right)$,

which does not depend on parametrization or gauge parameters. Let us note that the cancellation of the gauge and parametrization dependencies in this expression looks rather impressive, taking the (somehow scaring) form of the expressions in Appendix A.

The coefficient 17/20 does not agree with the value presented in Ref. [11]. This discordance is due to a missing factor of four in formula (35) of [11], as we explain in the Appendix B. It is remarkable that the complicated calculation in this work has only this small mistake and is otherwise correct.

\section{General proof of gauge independence}

We have evaluated the coefficients $\beta_{i}$ explicitly in the most general parametrization for the quantum field and the particular case of massive point-like masses. However, given Eq. (36), we provided an expression for the Newtonian potential (73) independently on the explicit values of the $\beta_{i}$. Therefore, to prove that the potential (73) does not depend on the gauge-fixing and parametrization choices, it is enough to prove that the following combination,

$\beta_{\text {inv }}=\frac{4}{3} \beta_{2}-\frac{1}{3} \beta_{0}-2 \beta_{R T 1}-2 \beta_{R T 2}+4 \beta_{T T}$,

is a gauge and parametrization invariant quantity. For this, we follow $[23,34,35]$, i.e., we employ the general statement about the gauge-fixing and parametrization independence of the on-shell effective action. In particular, the difference between the divergences of two versions of the oneloop effective action, evaluated using different gauge and parametrizations $\alpha_{i}$ and $\alpha_{0}$, is proportional to the classical equations of motion,

$$
\begin{aligned}
\delta \bar{\Gamma}_{d i v}^{(1)}= & \bar{\Gamma}_{d i v}^{(1)}\left(\alpha_{i}\right)-\bar{\Gamma}_{d i v}^{(1)}\left(\alpha_{0}\right)=-\frac{\mu^{n-4}}{(4 \pi)^{2}(n-4)} \\
& \times \int d^{n} x \sqrt{-g} \varepsilon^{\mu \nu} f_{\mu \nu}, \\
\varepsilon^{\mu \nu}= & R^{\mu \nu}-\frac{1}{2} g^{\mu \nu} R-\frac{\kappa^{2}}{2} T^{\mu \nu} .
\end{aligned}
$$

As the divergences are local and covariant quantities with mass dimension four, the tensor function $f_{\mu \nu}$ has the following general structure

$f_{\mu \nu}=b_{1} R_{\mu \nu}+b_{2} R g_{\mu \nu}+\kappa^{2} b_{3} T_{\mu \nu}+\kappa^{2} b_{4} T g_{\mu \nu}$,

where the parameters $b_{1,2,3,4}$ depend on the choice of the gauge and parametrization parameters $\alpha_{i}$. Thus, replacing (77) in (76) and using the definition (29) we get:

$$
\begin{aligned}
& \delta \bar{\Gamma}_{d i v}^{(1)}=-\frac{\mu^{n-4}}{(4 \pi)^{2}(n-4)} \int d^{n} x \sqrt{-g} \\
& \quad \times\left\{b_{1} R_{\mu \nu}^{2}-\left(\frac{1}{2} b_{1}+b_{2}\right) R^{2}+\kappa^{2}\left(b_{3}-\frac{1}{2} b_{1}\right) R_{\mu \nu} T^{\mu \nu}\right. \\
& \left.-\kappa^{2}\left(\frac{1}{2} b_{2}+\frac{1}{2} b_{3}+b_{4}\right) R T-\kappa^{4}\left(\frac{1}{2} b_{3}+\frac{1}{2} b_{4}\right) T^{2}\right\} .
\end{aligned}
$$

So, under a gauge and/or parametrization transformation, the coefficients in (31) transform according to:

$$
\begin{aligned}
& c_{1} \rightarrow c_{1}, \quad c_{2} \rightarrow c_{2}+b_{1}, \quad c_{3} \rightarrow c_{3}-\left(\frac{b_{1}}{2}+b_{2}\right), \\
& c_{4} \rightarrow c_{4}+\left(b_{3}-\frac{b_{1}}{2}\right), \\
& c_{5} \rightarrow c_{5}-\frac{1}{2}\left(b_{2}+b_{3}+2 b_{4}\right), \quad c_{6} \rightarrow c_{6}-\frac{1}{2}\left(b_{3}+b_{4}\right) .
\end{aligned}
$$


It is a simple exercise to check that, besides $c_{1}$, there is the following gauge- and parametrization-independent combination:

$c_{\text {inv }}=c_{2}+c_{3}+c_{4}-2 c_{5}+4 c_{6}$.

In particular, in the notations of (36), the formula (80) implies that (75) is truly gauge and parametrization invariant. This also means the physical results derived on the basis of usual effective action and for the unique Vilkovisky-DeWitt effective action [28] will be the same. The reader can consult [26] for the first calculation in quantum gravity in this formalism and the more recent Ref. [24] for the explicit verification of the parameterization independence, including the conformal mode (5).

In fact, the generalization of the calculations done in [24] to include the massive point particles is straightforward. Without entering in the full details, the result for the one-loop divergences in the theory (3), using the Vilkovisky-DeWitt definition for the effective action and the general parametrization (5), has the same form as Eq. (31), but now with the following gauge and parametrization independent coefficients,

$$
\begin{aligned}
& c_{1}=\frac{53}{45}, \quad c_{2}=-\frac{61}{90}, \quad c_{3}=\frac{25}{36}, \quad c_{4}=-\frac{3}{2}, \\
& c_{5}=\frac{1}{2}, \quad c_{6}=-\frac{5}{32} .
\end{aligned}
$$

Using (38) and (73), it is easy to verify that (81) provides the same Newtonian potential of Eq. (74). Thus, the cancellation observed in (74) is a necessary feature of effective quantum gravity, especially in the case when only metric is a quantum field, while the macroscopic massive bodies are treated as classical sources, according to the arguments of $[11,13]$.

As the massive sources are regarded classical, one can certainly expect the parametrization independence to hold for other configurations of matter, including non-point mass distributions, such as, e.g., dark matter.

\section{Universality of the effective field theory result}

The effective field theory approach cannot be taken lightly, as we cannot write down all possible action operators without having any guiding principle. Indeed, it is well known that any higher derivative theory has a number of ghost-like states depending on the number of derivatives that catastrophically make the vacuum decay instantaneously in ghost and normal particles. This is a trivial consequence of the energy conservation since ghosts carry negative energy [41]. Therefore, EFTs make sense and are well defined only if they turn out to be the low-energy approximation, or large distance limit, of more fundamental theories consistent with unitarity (perturbative), and (super-)renormalizability or finiteness.
One example of finite and unitarity theory is string theory, but we here focus on ultraviolet complete gravitational theories in the quantum field theory framework. In the latter case, we have two classes of well-defined theories: higher derivative theories à la Lee-Wick [42-46] or more general higher derivative theories [47], in which unitarity can be formally achieved by means of the Anselmi-Piva prescription, which is an extension of conventional Wick rotation, operational for the higher derivative models [48-50]; or nonlocal theories without ghost-like perturbative degrees of freedom [51-55]. In Lee-Wick quantum gravity, the ghost particles may only have complex mass square $[44,45]$, while in general higher derivative theories [47] we also have real ghosts, but in both cases, such states do not appear as asymptotic states. Indeed, such homogeneous solutions of the linear equations of motion can be removed from the spectrum of the theory by hand and, most importantly, they are not created again in the loop amplitudes once the Anselmi-Piva prescription is implemented. This is very similar to what happens with the BRST ghosts that appear because of the quantum gauge invariance. Indeed, we can safely fix the number of external BRST ghosts to zero, because in the loop amplitudes, they are exactly canceled by the non-physical polarization states of the gauge bosons. Similarly, in higher derivative theories, we can fix the number of external ghosts to zero because they are not regenerated in the loop amplitudes at any perturbative order whether the prescription [48-50] is implemented.

Differently, in nonlocal quantum gravity [51-55], the perturbative degrees of freedom are the same as the local theory $[60,61]$ and the convergence of the loop amplitudes is achieved by introducing an exponential (but asymptotically polynomial [51-53]) form factor that has no poles in the whole complex plane. At the quantum level, the loop amplitudes are computed in the Euclidean signature, and afterwards, the physical amplitudes are obtained using an analytic continuation of the external energies from the Euclidean space to Minkowski spacetime [56,57]. A unified nonlocal theory of all fundamental interactions with the aforementioned properties of the purely gravitational theories has been recently proposed in $[62,63]$. In the ultraviolet regime all nonlocal gravitational theories (or local with more than ten derivatives in four dimensions) are asymptotically free [58] or finite [54,59]. Theories with a number of derivatives from six to eight are super-renormalizable but, to prove asymptotic freedom, quantitative analysis and explicit computations are needed because there are divergences also at two and/or three loops. The last theory which deserves special attention is the four derivatives or Stelle's theory [66].

Said this, it is very interesting to investigate the infrared properties of any of the theories described above. In particular, in this work, we are interested in the leading correction to the Newtonian potential. Such correction is strictly related to the analytic properties of the one-loop amplitude regardless 
of the renormalizability or finiteness of the theory. Indeed, the analyticity only depends on the Landau singularities of the one-loop amplitude, namely when the denominators of the amplitude are zero for the same value of the external energy. This property depends only on the low-energy spectrum of the theory, and, hence, the contribution of the massive states is sub-dominant. Therefore, the main corrections to the quantum effective action at large distance come only from the massless states.

As an example we can consider a nonlocal scalar theory with an exponential form factor [64]. This theory is ultraviolet finite, but the one-loop amplitude at low energy, namely when $p^{2} / \Lambda^{2} \lesssim 1$ (here $\Lambda$ is the non-locality scale), shows up exactly the same logarithmic non-analyticity of the local theory. This result is understandable whether we identify $\Lambda$ with the cut-off of the local theory.

Let us expand on the case of the scalar model. The nonlocal Lagrangian that we would like to consider reads:

$\mathcal{L}_{\phi}=-\frac{1}{2} \phi \mathrm{e}^{H\left(-\square / \Lambda^{2}\right)}\left(\square+m^{2}\right) \phi-\frac{\lambda}{3 !} \phi^{3}$,

where $H(z)$ is an analytic function without poles in the complex plane at finite distance. The propagator of the scalar field is:

$G(k)=\frac{\mathrm{ie}^{-H(k)}}{k^{2}-m^{2}+\mathrm{i} \epsilon}$.

The one-loop amplitude turns out to be:

$\mathcal{M}=\frac{\lambda^{2}}{32 \pi^{4}} \int_{-\infty}^{+\infty} d^{4} k_{\mathrm{E}} \frac{\mathrm{e}^{-H\left(k_{\mathrm{E}}\right)} \mathrm{e}^{-H\left(k_{\mathrm{E}}-p_{\mathrm{E}}\right)}}{\left(k_{\mathrm{E}}^{2}+m^{2}\right)\left[\left(k_{\mathrm{E}}+p_{\mathrm{E}}\right)^{2}+m^{2}\right]}$,

where $k_{\mathrm{E}}$ and $p_{\mathrm{E}}$ are respectively the internal and external purely imaginary four-momenta. For the simplest nonlocality, $H=\square=-k^{2} / \Lambda^{2}=k_{\mathrm{E}}^{2} / \Lambda^{2}$, the above amplitude (84) turns into a simple Gaussian integral. The explicit computation was done for the first time in [64] and more recently in [65]. The result after the analytic continuation from purely imaginary to real external energy reads:

$\mathcal{M}=-\frac{\lambda^{2}}{16 \pi^{2}} \int_{0}^{1 / 2} d x \operatorname{Ei}\left[-\left(\frac{m^{2}-p^{2} x(1-x)}{x \Lambda^{2}}\right)\right]$,

where $\operatorname{Ei}(z)$ is the exponential integral. Notice that the result is finite without need of any regularization.

We now consider the low energy limit $p^{2} / \Lambda^{2} \lesssim 1$. In order to investigate such limit, we remind the Taylor expansion of the exponential integral $\operatorname{Ei}(z)$,

$\operatorname{Ei}(z)=\gamma_{\mathrm{E}}+\ln z+z+\frac{z^{2}}{4}+\cdots$.

Using the above expansion in (85) we get:

$\mathcal{M} \simeq-\frac{\lambda^{2}}{32 \pi^{2}} \int_{0}^{1} d x \ln \left(\frac{m^{2}-p^{2} x(1-x)}{\Lambda^{2}}\right)$.
Therefore, (85) at low energy resembles the result in the local quantum field theory up to proper identification of the nonlocality scale with the cut-off of the local renormalizable theory, which we give here for completeness:

$\Lambda^{2}=\Lambda_{\text {cut }- \text { off }} \mathrm{e}^{\gamma_{\mathrm{E}}+1+\ln 2}$.

As another example, let us now consider a nonlocal theory without perturbative degrees of freedom around the usual trivial background $\phi_{\mathrm{B}}=0$. The theory reads:

$\mathcal{L}_{\phi}=-\frac{1}{2} \phi \mathrm{e}^{H\left(-\square / \Lambda^{2}\right)} m^{2} \phi-\frac{\lambda}{3 !} \phi^{3}$.

Clearly, at the zero-order in $\lambda$ the perturbative solution of the free-theory equation of motion is $\phi=0$, namely

$\mathrm{e}^{H\left(-\square / \Lambda^{2}\right)} m^{2} \phi=0 \Longrightarrow \phi=0$.

Therefore, there are no perturbative degrees of freedom propagating around the trivial background. Equivalently, the propagator in momentum space, which can be obtained from (83) removing $k^{2}$ from the denominator, has no poles in the whole complex plane at finite energy,

$G(k)=-\frac{\mathrm{i}}{m^{2}} \mathrm{e}^{-H\left(k^{2} / \Lambda^{2}\right)}$,

consistently with the classical solution in (90). Now we are ready to compute one-loop bubble diagram for the theory (89). The one-loop amplitude reads:

$\mathcal{M}=-\frac{\lambda^{2}}{32 \pi^{4}} \int_{-\infty}^{+\infty} d^{4} k_{\mathrm{E}} \frac{\mathrm{i} \mathrm{e}^{-H\left(k_{\mathrm{E}}\right)}}{m^{2}} \frac{\mathrm{i} \mathrm{e}^{-H\left(k_{\mathrm{E}}-p_{\mathrm{E}}\right)}}{m^{2}}$.

Again for the case of a Gaussian non-locality, namely $H=$ $\square+m^{2}=\left(-k^{2}+m^{2}\right) / \Lambda^{2}=\left(k_{\mathrm{E}}^{2}+m^{2}\right) / \Lambda^{2}$, the amplitude (92) simplifies to:

$$
\begin{aligned}
\mathcal{M} & =-\frac{\lambda^{2}}{32 \pi^{4}} \int_{-\infty}^{+\infty} d^{4} k_{\mathrm{E}} \frac{\mathrm{i} \mathrm{e}^{-\frac{k_{\mathrm{E}}^{2}+m^{2}}{\Lambda^{2}}}}{m^{2}} \frac{\mathrm{ie}^{-\frac{\left(k_{\mathrm{E}}-p_{\mathrm{E}}\right)^{2}+m^{2}}{\Lambda^{2}}}}{m^{2}} \\
& =\frac{\lambda^{2}}{128 \pi^{2}} \mathrm{e}^{-\frac{1}{2 \Lambda^{2}}\left(-p^{2}+(2 m)^{2}\right)},
\end{aligned}
$$

which is analytic in the whole complex plane according to the absence of perturbative degrees of freedom in the theory (89).

We conclude that the logarithm correction to the quantum effective action is universally related to the low energy spectrum of the theory regardless of the ultraviolet completion of the theory.

The same argument applies to a general nonlocal or higher derivative theory, being finite or (super-)renormalizable, with the graviton the only massless state in the spectrum. The action for a general local higher derivative gravitational theory reads:

$S_{\mathrm{HD}}=\int d^{D} x \sqrt{-g}\left[\kappa^{-2} R+\sum_{n=0}^{\mathrm{N}}\left(\omega_{0, n} R \square^{n} R+\omega_{2, n} R_{\mu \nu} \square^{n} R^{\mu \nu}\right)\right.$ 


$$
\left.+V\left(\mathcal{O}\left(\operatorname{Riem}^{3}\right)\right)\right]
$$

where the last term plays the role of a potential because it is at least cubic in the Riemann tensor and, then, it does not contribute to the propagator around the Minkowski's background. Moreover, the potential contains at most $2 \mathrm{~N}+4$ derivatives in order to not spoil the super-renormalizability of the theory. However, the presence of $V\left(\mathcal{O}\left(\mathrm{Riem}^{3}\right)\right)$ is crucial to making the theory finite $[53,54]$. The particle spectrum of the higher derivative action (94) contains a massless graviton and a finite number of massive states (including complex and real ghosts) whose masses are defined in terms of the constants $\kappa, \omega_{0, n}$, and $\omega_{2, n}$. As said above, the theory (94) can be finite, namely all the loop amplitudes are convergent. However, at an energy scale much smaller than the mass of all the massive states, the one-loop quantum effective action has the same form as (40) for a proper identification (which means up to an overall dimensionless rescaling) of the renormalization group invariant scale $\mu$ in (40) with the mass scale that is implicitly hidden in the parameters $\omega_{0, n}$ and $\omega_{2, n}$. In other words, at low energy, the higher derivative operators play the role of a higher derivative regularization. Once again, this is not surprising because they are related to the analytic properties of the one-loop quantum effective action that only depends on the Landau singularities of the amplitude. Moreover, in the low energy limit the contribution of the massive states is sub-leading with respect to the massless graviton.

\section{Conclusions and discussions}

We have reconsidered the arguments for taking into account only the gravitational loops in effective quantum gravity. The physical reasons and, in particular, the analogy with the Casimir force show that the massive sources should not be quantized. Taking this into account, it is well-known that the numerical effects of quantum corrections are too small to be measured in the laboratory. However, this does not make irrelevant the consistent derivation of the quantum corrections and the discussion of their (non)universality.

The main part of the work reports on a calculation similar to the previously done in [11], but in an arbitrary parametrization of quantum perturbation of the metric. As a result, we have found that the effective quantum gravity correction to Newton's gravitational potential is completely independent of the choice of the parametrization. Together with the mentioned qualitative arguments and the gauge-fixing independence established in [11], our result ensures that the final outcome (73) is correct. In principle, this result can be generalized for more complicated distributions of mass. In this respect, the more general proof of invariance given in Sect. 5 is relevant, as it provides a good expectation to obtain sound results.

Furthermore, we discussed in Sect. 6 the universality and independence of the leading correction to Newton's potential in the effective field theories resulting in the low-energy limit of ultraviolet complete theories of quantum gravity in the quantum field theory framework. In particular, we showed that the leading quantum correction to Newton's potential is strictly related to the low-energy spectrum of the fundamental theory rather than the details of the ultraviolet complete quantum gravity theory, in accordance with the general effective quantum field theory approach. Indeed, the logarithm quantum correction is related to the Landau singularities and the analytic properties of the one-loop amplitude, which only depend on the low-energy spectrum of the classical theory. Therefore, not all the quantum effective theories produce the same result. The latter statement has been shown explicitly with simple examples.

Taking the parametrization and gauge invariance, the direct relation to the beta functions, and the fact that the quantum corrections are the same as in the usual and VilkoviskyDeWitt versions of the effective actions, the main result for the quantum corrected potential can be compared to the invariant version of the renormalization group improved classical action [36,37]. In the present framework, when dealing with the Newtonian interaction between two massive particles, the interpretation of the renormalization group scale is straightforward. This interpretation is much more subtle and complicated in other physical situations, e.g., in Cosmology [38], where some specially designed procedures can be applied $[39,40]$, but still do not guarantee unique interpretation of the scale. Thus, it would be interesting to compare the two ways of scale identification in the cases like the one considered above, with a well-defined procedure [11].

In conclusion, let us note that the main approximation in effective quantum gravity is supposed to hold between the Planck scale in the UV and the Hubble scale in the IR . In particular, this interval includes all the physically and astronomically interesting high-energy gravitational phenomena related to early cosmology and black hole physics. Thus, there still is a possibility to find applications to the results based on correctly defined quantum gravity corrections to classical gravity.

Acknowledgements Authors are grateful to Michael Bordag, Michael Duff and Diego Mazzitelli for useful and friendly discussions. I.Sh. was partially supported by Conselho Nacional de Desenvolvimento Científico e Tecnológico - CNPq (Brazil) under the grant 303635/20185, by Fundação de Amparo à Pesquisa de Minas Gerais - FAPEMIG under the project PPM-00604-18, and by the Ministry of Education of Russian Federation under the project No. FEWF-2020-0003. L.M. was supported by the Basic Research Program of Science, Technology and Innovation Commission of Shenzhen Municipality (Grant No. JCYJ20180302174206969). 
Data Availability Statement The manuscript has associated data in a data repository. [Authors' comment: All data included in this manuscript are available upon request by contacting with the corresponding author.]

Open Access This article is licensed under a Creative Commons Attribution 4.0 International License, which permits use, sharing, adaptation, distribution and reproduction in any medium or format, as long as you give appropriate credit to the original author(s) and the source, provide a link to the Creative Commons licence, and indicate if changes were made. The images or other third party material in this article are included in the article's Creative Commons licence, unless indicated otherwise in a credit line to the material. If material is not included in the article's Creative Commons licence and your intended use is not permitted by statutory regulation or exceeds the permitted use, you will need to obtain permission directly from the copyright holder. To view a copy of this licence, visit http://creativecomm ons.org/licenses/by/4.0/.

Funded by SCOAP ${ }^{3}$.

\section{Appendix A}

The explicit expressions for the coefficients in (31) were obtained using WolfRAm MATHEMATICA [67] and the auxiliary tensor algebra package XACT $[68,69]$. For the sake of completeness, we present the list of the coefficients used in Eq. (32):

$$
\begin{aligned}
\xi_{2}= & 16 \lambda^{2} \gamma_{0}^{2} \gamma_{1}^{4}+8 \lambda \gamma_{0} \gamma_{1}^{3} \gamma_{3} \\
& -128 \lambda^{2} \gamma_{0}^{2} \gamma_{3}^{2}-32 \lambda \gamma_{0} \gamma_{1} \gamma_{3}^{2}-\gamma_{1}^{2} \gamma_{3}^{2} \\
& -128 \lambda \gamma_{0} \gamma_{2} \gamma_{3}^{2}-16 \gamma_{1} \gamma_{2} \gamma_{3}^{2} \\
& -32 \gamma_{2}^{2} \gamma_{3}^{2}+16 \lambda \gamma_{0} \gamma_{1}^{3} \gamma_{5}+4 \gamma_{1}^{2} \gamma_{3} \gamma_{5} \\
& +4 \gamma_{1}^{2} \gamma_{5}^{2} \\
\xi_{3}= & 3584 \lambda^{4} \gamma_{0}^{4} \gamma_{1}^{4}+1024 \lambda^{3} \gamma_{0}^{3} \gamma_{1}^{5}+64 \lambda^{2} \gamma_{0}^{2} \gamma_{1}^{6} \\
& -4 \lambda \gamma_{0} \gamma_{1}^{7}+4096 \lambda^{3} \gamma_{0}^{3} \gamma_{1}^{4} \gamma_{2}+512 \lambda^{2} \gamma_{0}^{2} \gamma_{1}^{5} \gamma_{2} \\
& -48 \lambda \gamma_{0} \gamma_{1}^{6} \gamma_{2}+1024 \lambda^{2} \gamma_{0}^{2} \gamma_{1}^{4} \gamma_{2}^{2} \\
& -192 \lambda \gamma_{0} \gamma_{1}^{5} \gamma_{2}^{2}-256 \lambda \gamma_{0} \gamma_{1}^{4} \gamma_{2}^{3} \\
& -55296 \lambda^{4} \gamma_{0}^{4} \gamma_{1}^{2} \gamma_{3} \\
& -26112 \lambda^{3} \gamma_{0}^{3} \gamma_{1}^{3} \gamma_{3}-4736 \lambda^{2} \gamma_{0}^{2} \gamma_{1}^{4} \gamma_{3} \\
& -392 \lambda \gamma_{0} \gamma_{1}^{5} \gamma_{3}-14 \gamma_{1}^{6} \gamma_{3}-110592 \lambda^{3} \gamma_{0}^{3} \gamma_{1}^{2} \gamma_{2} \gamma_{3} \\
& -39936 \lambda^{2} \gamma_{0}^{2} \gamma_{1}^{3} \gamma_{2} \gamma_{3} \\
& -4928 \lambda \gamma_{0} \gamma_{1}^{4} \gamma_{2} \gamma_{3}-220 \gamma_{1}^{5} \gamma_{2} \gamma_{3} \\
& -82944 \lambda^{2} \gamma_{0}^{2} \gamma_{1}^{2} \gamma_{2}^{2} \gamma_{3} \\
& -20352 \lambda \gamma_{0} \gamma_{1}^{3} \gamma_{2}^{2} \gamma_{3}-1304 \gamma_{1}^{4} \gamma_{2}^{2} \gamma_{3} \\
& -27648 \lambda \gamma_{0} \gamma_{1}^{2} \gamma_{2}^{3} \gamma_{3} \\
& -3456 \gamma_{1}^{3} \gamma_{2}^{3} \gamma_{3} \\
& -3456 \gamma_{1}^{2} \gamma_{2}^{4} \gamma_{3}+30720 \lambda^{4} \gamma_{0}^{4} \gamma_{3}^{2} \\
+ & 15360 \lambda^{3} \gamma_{0}^{3} \gamma_{1} \gamma_{3}^{2}+3072 \lambda^{2} \gamma_{0}^{2} \gamma_{1}^{2} \gamma_{3}^{2} \\
+ & 288 \lambda \gamma_{0} \gamma_{1}^{3} \gamma_{3}^{2} \\
+ & 12 \gamma_{1}^{4} \gamma_{3}^{2}+61440 \lambda^{3} \gamma_{0}^{3} \gamma_{2} \gamma_{3}^{2} \\
& -61
\end{aligned}
$$

$$
\begin{aligned}
& +23040 \lambda^{2} \gamma_{0}^{2} \gamma_{1} \gamma_{2} \gamma_{3}^{2}+3072 \lambda \gamma_{0} \gamma_{1}^{2} \gamma_{2} \gamma_{3}^{2} \\
& +144 \gamma_{1}^{3} \gamma_{2} \gamma_{3}^{2} \\
& +46080 \lambda^{2} \gamma_{0}^{2} \gamma_{2}^{2} \gamma_{3}^{2}+11520 \lambda \gamma_{0} \gamma_{1} \gamma_{2}^{2} \gamma_{3}^{2} \\
& +768 \gamma_{1}^{2} \gamma_{2}^{2} \gamma_{3}^{2}+15360 \lambda \gamma_{0} \gamma_{2}^{3} \gamma_{3}^{2} \\
& +1920 \gamma_{1} \gamma_{2}^{3} \gamma_{3}^{2} \\
& +1920 \gamma_{2}^{4} \gamma_{3}^{2}-221184 \lambda^{4} \gamma_{0}^{4} \gamma_{1}^{2} \gamma_{4} \\
& -110592 \lambda^{3} \gamma_{0}^{3} \gamma_{1}^{3} \gamma_{4}-20480 \lambda^{2} \gamma_{0}^{2} \gamma_{1}^{4} \gamma_{4} \\
& -1664 \lambda \gamma_{0} \gamma_{1}^{5} \gamma_{4} \\
& -56 \gamma_{1}^{6} \gamma_{4}-442368 \lambda^{3} \gamma_{0}^{3} \gamma_{1}^{2} \gamma_{2} \gamma_{4} \\
& -165888 \lambda^{2} \gamma_{0}^{2} \gamma_{1}^{3} \gamma_{2} \gamma_{4}-20480 \lambda \gamma_{0} \gamma_{1}^{4} \gamma_{2} \gamma_{4} \\
& -880 \gamma_{1}^{5} \gamma_{2} \gamma_{4}-331776 \lambda^{2} \gamma_{0}^{2} \gamma_{1}^{2} \gamma_{2}^{2} \gamma_{4} \\
& -82944 \lambda \gamma_{0} \gamma_{1}^{3} \gamma_{2}^{2} \gamma_{4}-5216 \gamma_{1}^{4} \gamma_{2}^{2} \gamma_{4}+768 \lambda^{2} \gamma_{0}^{2} \gamma_{1}^{2} \gamma_{5}^{2} \\
& -110592 \lambda \gamma_{0} \gamma_{1}^{2} \gamma_{2}^{3} \gamma_{4}-13824 \gamma_{1}^{3} \gamma_{2}^{3} \gamma_{4} \\
& -13824 \gamma_{1}^{2} \gamma_{2}^{4} \gamma_{4}+442368 \lambda^{4} \gamma_{0}^{4} \gamma_{3} \gamma_{4} \\
& +221184 \lambda^{3} \gamma_{0}^{3} \gamma_{1} \gamma_{3} \gamma_{4}+41472 \lambda^{2} \gamma_{0}^{2} \gamma_{1}^{2} \gamma_{3} \gamma_{4} \\
& +3456 \lambda \gamma_{0} \gamma_{1}^{3} \gamma_{3} \gamma_{4}+120 \gamma_{1}^{4} \gamma_{3} \gamma_{4} \\
& +884736 \lambda^{3} \gamma_{0}^{3} \gamma_{2} \gamma_{3} \gamma_{4}+331776 \lambda^{2} \gamma_{0}^{2} \gamma_{1} \gamma_{2} \gamma_{3} \gamma_{4} \\
& +41472 \lambda \gamma_{0} \gamma_{1}^{2} \gamma_{2} \gamma_{3} \gamma_{4} \\
& +1728 \gamma_{1}^{3} \gamma_{2} \gamma_{3} \gamma_{4}+663552 \lambda^{2} \gamma_{0}^{2} \gamma_{2}^{2} \gamma_{3} \gamma_{4} \\
& +165888 \lambda \gamma_{0} \gamma_{1} \gamma_{2}^{2} \gamma_{3} \gamma_{4}+10368 \gamma_{1}^{2} \gamma_{2}^{2} \gamma_{3} \gamma_{4} \\
& +221184 \lambda \gamma_{0} \gamma_{2}^{3} \gamma_{3} \gamma_{4} \\
& +27648 \gamma_{1} \gamma_{2}^{3} \gamma_{3} \gamma_{4}+27648 \gamma_{2}^{4} \gamma_{3} \gamma_{4} \\
& +884736 \lambda^{4} \gamma_{0}^{4} \gamma_{4}^{2} \\
& +82944 \lambda^{2} \gamma_{0}^{2} \gamma_{1}^{2} \gamma_{4}^{2}+6912 \lambda \gamma_{0} \gamma_{1}^{3} \gamma_{4}^{2}+240 \gamma_{1}^{4} \gamma_{4}^{2} \\
& +1769472 \lambda^{3} \gamma_{0}^{3} \gamma_{2} \gamma_{4}^{2} \\
& +82944 \lambda \gamma_{0} \gamma_{1}^{2} \gamma_{2} \gamma_{4}^{2}+3456 \gamma_{1}^{3} \gamma_{2} \gamma_{4}^{2} \\
& +1327104 \lambda^{2} \gamma_{0}^{2} \gamma_{2}^{2} \gamma_{4}^{2}+331776 \lambda \gamma_{0} \gamma_{1} \gamma_{2}^{2} \gamma_{4}^{2} \\
& +20736 \gamma_{1}^{2} \gamma_{2}^{2} \gamma_{4}^{2}+442368 \lambda \gamma_{0} \gamma_{2}^{3} \gamma_{4}^{2} \\
& +55296 \gamma_{1} \gamma_{2}^{3} \gamma_{4}^{2}+55296 \gamma_{2}^{4} \gamma_{4}^{2} \\
& +3072 \lambda^{3} \gamma_{0}^{3} \gamma_{1}^{3} \gamma_{5} \\
& +1024 \lambda^{2} \gamma_{0}^{2} \gamma_{1}^{4} \gamma_{5}+112 \lambda \gamma_{0} \gamma_{1}^{5} \gamma_{5}-2 \gamma_{1}^{6} \gamma_{5} \\
& +3072 \lambda^{2} \gamma_{0}^{2} \gamma_{1}^{3} \gamma_{2} \gamma_{5}+640 \lambda \gamma_{0} \gamma_{1}^{4} \gamma_{2} \gamma_{5} \\
& -16 \gamma_{1}^{5} \gamma_{2} \gamma_{5} \\
& +768 \lambda \gamma_{0} \gamma_{1}^{3} \gamma_{2}^{2} \gamma_{5}-32 \gamma_{1}^{4} \gamma_{2}^{2} \gamma_{5} \\
& +768 \lambda^{2} \gamma_{0}^{2} \gamma_{1}^{2} \gamma_{3} \gamma_{5}+48 \gamma_{1}^{4} \gamma_{4} \gamma_{5} \\
& +192 \lambda \gamma_{0} \gamma_{1}^{3} \gamma_{3} \gamma_{5}+24 \gamma_{1}^{4} \gamma_{3} \gamma_{5} \\
& +768 \lambda \gamma_{0} \gamma_{1}^{2} \gamma_{2} \gamma_{3} \gamma_{5}+96 \gamma_{1}^{3} \gamma_{2} \gamma_{3} \gamma_{5} \\
& +192 \gamma_{1}^{2} \gamma_{2}^{2} \gamma_{3} \gamma_{5} \\
& +192 \lambda \gamma_{0} \gamma_{1}^{3} \gamma_{5}^{2}+36 \gamma_{1}^{4} \gamma_{5}^{2}
\end{aligned}
$$




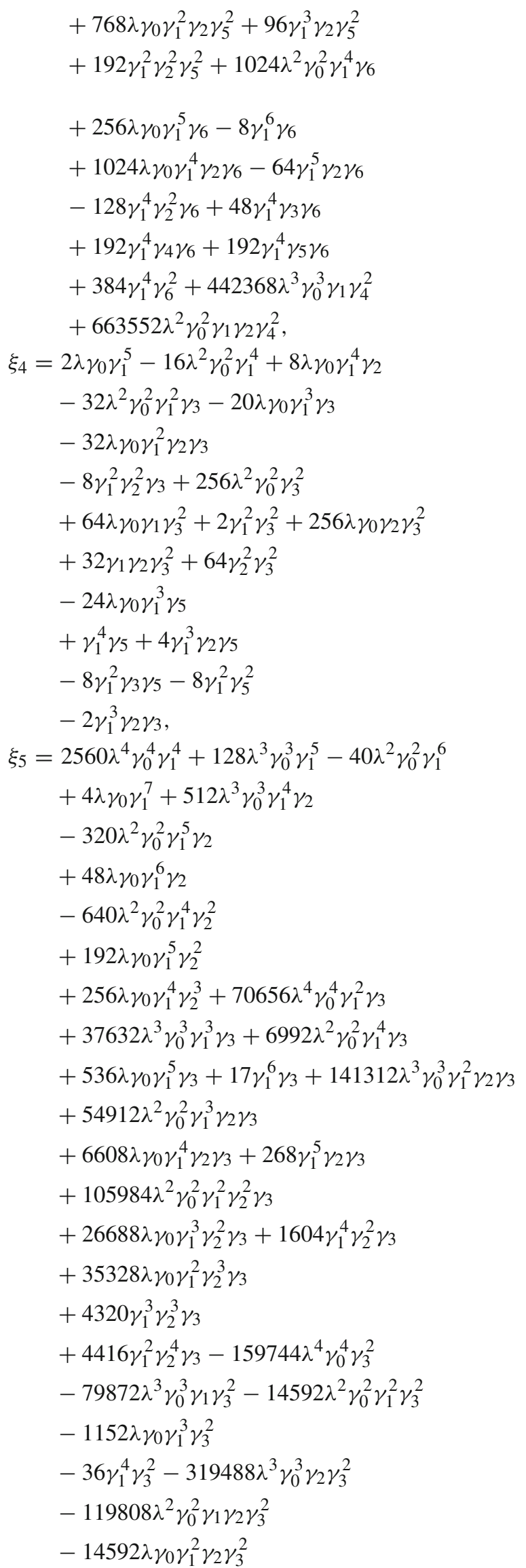

$-576 \gamma_{1}^{3} \gamma_{2} \gamma_{3}^{2}-239616 \lambda^{2} \gamma_{0}^{2} \gamma_{2}^{2} \gamma_{3}^{2}$

$-59904 \lambda \gamma_{0} \gamma_{1} \gamma_{2}^{2} \gamma_{3}^{2}$

$-3648 \gamma_{1}^{2} \gamma_{2}^{2} \gamma_{3}^{2}$

$-79872 \lambda \gamma_{0} \gamma_{2}^{3} \gamma_{3}^{2}-9984 \gamma_{1} \gamma_{2}^{3} \gamma_{3}^{2}$

$-9984 \gamma_{2}^{4} \gamma_{3}^{2}+258048 \lambda^{4} \gamma_{0}^{4} \gamma_{1}^{2} \gamma_{4}$

$+129024 \lambda^{3} \gamma_{0}^{3} \gamma_{1}^{3} \gamma_{4}$

$+23744 \lambda^{2} \gamma_{0}^{2} \gamma_{1}^{4} \gamma_{4}+1904 \lambda \gamma_{0} \gamma_{1}^{5} \gamma_{4}$

$+68 \gamma_{1}^{6} \gamma_{4}+516096 \lambda^{3} \gamma_{0}^{3} \gamma_{1}^{2} \gamma_{2} \gamma_{4}$

$+193536 \lambda^{2} \gamma_{0}^{2} \gamma_{1}^{3} \gamma_{2} \gamma_{4}+23744 \lambda \gamma_{0} \gamma_{1}^{4} \gamma_{2} \gamma_{4}$

$+1048 \gamma_{1}^{5} \gamma_{2} \gamma_{4}+387072 \lambda^{2} \gamma_{0}^{2} \gamma_{1}^{2} \gamma_{2}^{2} \gamma_{4}$

$+96768 \lambda \gamma_{0} \gamma_{1}^{3} \gamma_{2}^{2} \gamma_{4}+6128 \gamma_{1}^{4} \gamma_{2}^{2} \gamma_{4}$

$+129024 \lambda \gamma_{0} \gamma_{1}^{2} \gamma_{2}^{3} \gamma_{4}$

$+16128 \gamma_{1}^{3} \gamma_{2}^{3} \gamma_{4}$

$+16128 \gamma_{1}^{2} \gamma_{2}^{4} \gamma_{4}-884736 \lambda^{4} \gamma_{0}^{4} \gamma_{3} \gamma_{4}$

$-442368 \lambda^{3} \gamma_{0}^{3} \gamma_{1} \gamma_{3} \gamma_{4}$

$-82944 \lambda^{2} \gamma_{0}^{2} \gamma_{1}^{2} \gamma_{3} \gamma_{4}$

$-6912 \lambda \gamma_{0} \gamma_{1}^{3} \gamma_{3} \gamma_{4}-240 \gamma_{1}^{4} \gamma_{3} \gamma_{4}$

$-1769472 \lambda^{3} \gamma_{0}^{3} \gamma_{2} \gamma_{3} \gamma_{4}$

$-663552 \lambda^{2} \gamma_{0}^{2} \gamma_{1} \gamma_{2} \gamma_{3} \gamma_{4}$

$-82944 \lambda \gamma_{0} \gamma_{1}^{2} \gamma_{2} \gamma_{3} \gamma_{4}$

$-3456 \gamma_{1}^{3} \gamma_{2} \gamma_{3} \gamma_{4}-1327104 \lambda^{2} \gamma_{0}^{2} \gamma_{2}^{2} \gamma_{3} \gamma_{4}$

$+384 \lambda \gamma_{0} \gamma_{1}^{3} \gamma_{3} \gamma_{5}$

$-331776 \lambda \gamma_{0} \gamma_{1} \gamma_{2}^{2} \gamma_{3} \gamma_{4}$

$-20736 \gamma_{1}^{2} \gamma_{2}^{2} \gamma_{3} \gamma_{4}-442368 \lambda \gamma_{0} \gamma_{2}^{3} \gamma_{3} \gamma_{4}$

$+1536 \lambda^{2} \gamma_{0}^{2} \gamma_{1}^{2} \gamma_{3} \gamma_{5}$

$-55296 \gamma_{1} \gamma_{2}^{3} \gamma_{3} \gamma_{4}$

$-55296 \gamma_{2}^{4} \gamma_{3} \gamma_{4}$

$-1769472 \lambda^{4} \gamma_{0}^{4} \gamma_{4}^{2}$

$-884736 \lambda^{3} \gamma_{0}^{3} \gamma_{1} \gamma_{4}^{2}$

$-165888 \lambda^{2} \gamma_{0}^{2} \gamma_{1}^{2} \gamma_{4}^{2}-13824 \lambda \gamma_{0} \gamma_{1}^{3} \gamma_{4}^{2}$

$-480 \gamma_{1}^{4} \gamma_{4}^{2}$

$-3538944 \lambda^{3} \gamma_{0}^{3} \gamma_{2} \gamma_{4}^{2}$

$-1327104 \lambda^{2} \gamma_{0}^{2} \gamma_{1} \gamma_{2} \gamma_{4}^{2}-165888 \lambda \gamma_{0} \gamma_{1}^{2} \gamma_{2} \gamma_{4}^{2}$

$-6912 \gamma_{1}^{3} \gamma_{2} \gamma_{4}^{2}$

$-2654208 \lambda^{2} \gamma_{0}^{2} \gamma_{2}^{2} \gamma_{4}^{2}$

$-663552 \lambda \gamma_{0} \gamma_{1} \gamma_{2}^{2} \gamma_{4}^{2}-41472 \gamma_{1}^{2} \gamma_{2}^{2} \gamma_{4}^{2}$

$-884736 \lambda \gamma_{0} \gamma_{2}^{3} \gamma_{4}^{2}-110592 \gamma_{1} \gamma_{2}^{3} \gamma_{4}^{2}$

$-110592 \gamma_{2}^{4} \gamma_{4}^{2}+4608 \lambda^{3} \gamma_{0}^{3} \gamma_{1}^{3} \gamma_{5}$

$+512 \lambda^{2} \gamma_{0}^{2} \gamma_{1}^{4} \gamma_{5}-88 \lambda \gamma_{0} \gamma_{1}^{5} \gamma_{5}$ 


$$
\begin{aligned}
& +2 \gamma_{1}^{6} \gamma_{5} \\
& +3840 \lambda^{2} \gamma_{0}^{2} \gamma_{1}^{3} \gamma_{2} \gamma_{5}-256 \lambda \gamma_{0} \gamma_{1}^{4} \gamma_{2} \gamma_{5} \\
& +4 \gamma_{1}^{5} \gamma_{2} \gamma_{5}+384 \lambda \gamma_{0} \gamma_{1}^{3} \gamma_{2}^{2} \gamma_{5} \\
& -64 \gamma_{1}^{4} \gamma_{2}^{2} \gamma_{5} \\
& +1536 \lambda \gamma_{0} \gamma_{1}^{2} \gamma_{2} \gamma_{3} \gamma_{5}+192 \gamma_{1}^{3} \gamma_{2} \gamma_{3} \gamma_{5} \\
& +384 \gamma_{1}^{2} \gamma_{2}^{2} \gamma_{3} \gamma_{5} \\
& -96 \gamma_{1}^{4} \gamma_{4} \gamma_{5}-192 \gamma_{1}^{3} \gamma_{2}^{3} \gamma_{5} \\
& +1536 \lambda^{2} \gamma_{0}^{2} \gamma_{1}^{2} \gamma_{5}^{2} \\
& +384 \lambda \gamma_{0} \gamma_{1}^{3} \gamma_{5}^{2}-24 \gamma_{1}^{4} \gamma_{5}^{2} \\
& +1536 \lambda \gamma_{0} \gamma_{1}^{2} \gamma_{2} \gamma_{5}^{2}+192 \gamma_{1}^{3} \gamma_{2} \gamma_{5}^{2} \\
& +384 \gamma_{1}^{2} \gamma_{2}^{2} \gamma_{5}^{2}-1792 \lambda^{2} \gamma_{0}^{2} \gamma_{1}^{4} \gamma_{6} \\
& -448 \lambda \gamma_{0} \gamma_{1}^{5} \gamma_{6}+20 \gamma_{1}^{6} \gamma_{6}-1792 \lambda \gamma_{0} \gamma_{1}^{4} \gamma_{2} \gamma_{6} \\
& +160 \gamma_{1}^{5} \gamma_{2} \gamma_{6} \\
& +320 \gamma_{1}^{4} \gamma_{2}^{2} \gamma_{6}-96 \gamma_{1}^{4} \gamma_{3} \gamma_{6}-384 \gamma_{1}^{4} \gamma_{4} \gamma_{6} \\
& -384 \gamma_{1}^{4} \gamma_{5} \gamma_{6}-768 \gamma_{1}^{4} \gamma_{6}^{2} \text {, } \\
& \xi_{6}=-384 \lambda^{3} \gamma_{0}^{3} \gamma_{1}^{5}-88 \lambda^{2} \gamma_{0}^{2} \gamma_{1}^{6}-4 \lambda \gamma_{0} \gamma_{1}^{7} \\
& -1536 \lambda^{3} \gamma_{0}^{3} \gamma_{1}^{4} \gamma_{2}-704 \lambda^{2} \gamma_{0}^{2} \gamma_{1}^{5} \gamma_{2} \\
& -48 \lambda \gamma_{0} \gamma_{1}^{6} \gamma_{2} \\
& -1408 \lambda^{2} \gamma_{0}^{2} \gamma_{1}^{4} \gamma_{2}^{2}-192 \lambda \gamma_{0} \gamma_{1}^{5} \gamma_{2}^{2} \\
& -256 \lambda \gamma_{0} \gamma_{1}^{4} \gamma_{2}^{3}+9216 \lambda^{4} \gamma_{0}^{4} \gamma_{1}^{2} \gamma_{3} \\
& +5376 \lambda^{3} \gamma_{0}^{3} \gamma_{1}^{3} \gamma_{3} \\
& +944 \lambda^{2} \gamma_{0}^{2} \gamma_{1}^{4} \gamma_{3} \\
& +56 \lambda \gamma_{0} \gamma_{1}^{5} \gamma_{3}+\gamma_{1}^{6} \gamma_{3} \\
& +18432 \lambda^{3} \gamma_{0}^{3} \gamma_{1}^{2} \gamma_{2} \gamma_{3} \\
& +7296 \lambda^{2} \gamma_{0}^{2} \gamma_{1}^{3} \gamma_{2} \gamma_{3} \\
& +752 \lambda \gamma_{0} \gamma_{1}^{4} \gamma_{2} \gamma_{3}+20 \gamma_{1}^{5} \gamma_{2} \gamma_{3} \\
& +13824 \lambda^{2} \gamma_{0}^{2} \gamma_{1}^{2} \gamma_{2}^{2} \gamma_{3}+3264 \lambda \gamma_{0} \gamma_{1}^{3} \gamma_{2}^{2} \gamma_{3} \\
& +148 \gamma_{1}^{4} \gamma_{2}^{2} \gamma_{3} \\
& +4608 \lambda \gamma_{0} \gamma_{1}^{2} \gamma_{2}^{3} \gamma_{3} \\
& +480 \gamma_{1}^{3} \gamma_{2}^{3} \gamma_{3}+576 \gamma_{1}^{2} \gamma_{2}^{4} \gamma_{3}-43008 \lambda^{4} \gamma_{0}^{4} \gamma_{3}^{2} \\
& -21504 \lambda^{3} \gamma_{0}^{3} \gamma_{1} \gamma_{3}^{2}-3840 \lambda^{2} \gamma_{0}^{2} \gamma_{1}^{2} \gamma_{3}^{2} \\
& -288 \lambda \gamma_{0} \gamma_{1}^{3} \gamma_{3}^{2}-8 \gamma_{1}^{4} \gamma_{3}^{2} \\
& -86016 \lambda^{3} \gamma_{0}^{3} \gamma_{2} \gamma_{3}^{2} \\
& -32256 \lambda^{2} \gamma_{0}^{2} \gamma_{1} \gamma_{2} \gamma_{3}^{2}-3840 \lambda \gamma_{0} \gamma_{1}^{2} \gamma_{2} \gamma_{3}^{2} \\
& -144 \gamma_{1}^{3} \gamma_{2} \gamma_{3}^{2} \\
& -64512 \lambda^{2} \gamma_{0}^{2} \gamma_{2}^{2} \gamma_{3}^{2} \\
& -16128 \lambda \gamma_{0} \gamma_{1} \gamma_{2}^{2} \gamma_{3}^{2}-960 \gamma_{1}^{2} \gamma_{2}^{2} \gamma_{3}^{2} \\
& -21504 \lambda \gamma_{0} \gamma_{2}^{3} \gamma_{3}^{2}-2688 \gamma_{1} \gamma_{2}^{3} \gamma_{3}^{2}-2688 \gamma_{2}^{4} \gamma_{3}^{2} \\
& +12288 \lambda^{4} \gamma_{0}^{4} \gamma_{1}^{2} \gamma_{4}+6144 \lambda^{3} \gamma_{0}^{3} \gamma_{1}^{3} \gamma_{4} \\
& +1088 \lambda^{2} \gamma_{0}^{2} \gamma_{1}^{4} \gamma_{4}+80 \lambda \gamma_{0} \gamma_{1}^{5} \gamma_{4}+4 \gamma_{1}^{6} \gamma_{4} \\
& +24576 \lambda^{3} \gamma_{0}^{3} \gamma_{1}^{2} \gamma_{2} \gamma_{4} \\
& +9216 \lambda^{2} \gamma_{0}^{2} \gamma_{1}^{3} \gamma_{2} \gamma_{4}+1088 \lambda \gamma_{0} \gamma_{1}^{4} \gamma_{2} \gamma_{4} \\
& +56 \gamma_{1}^{5} \gamma_{2} \gamma_{4} \\
& +18432 \lambda^{2} \gamma_{0}^{2} \gamma_{1}^{2} \gamma_{2}^{2} \gamma_{4} \\
& +4608 \lambda \gamma_{0} \gamma_{1}^{3} \gamma_{2}^{2} \gamma_{4}+304 \gamma_{1}^{4} \gamma_{2}^{2} \gamma_{4} \\
& +6144 \lambda \gamma_{0} \gamma_{1}^{2} \gamma_{2}^{3} \gamma_{4} \\
& +768 \gamma_{1}^{3} \gamma_{2}^{3} \gamma_{4}+768 \gamma_{1}^{2} \gamma_{2}^{4} \gamma_{4} \\
& -147456 \lambda^{4} \gamma_{0}^{4} \gamma_{3} \gamma_{4}-73728 \lambda^{3} \gamma_{0}^{3} \gamma_{1} \gamma_{3} \gamma_{4} \\
& -13824 \lambda^{2} \gamma_{0}^{2} \gamma_{1}^{2} \gamma_{3} \gamma_{4}-1152 \lambda \gamma_{0} \gamma_{1}^{3} \gamma_{3} \gamma_{4} \\
& -40 \gamma_{1}^{4} \gamma_{3} \gamma_{4} \\
& -294912 \lambda^{3} \gamma_{0}^{3} \gamma_{2} \gamma_{3} \gamma_{4} \\
& -110592 \lambda^{2} \gamma_{0}^{2} \gamma_{1} \gamma_{2} \gamma_{3} \gamma_{4} \\
& -13824 \lambda \gamma_{0} \gamma_{1}^{2} \gamma_{2} \gamma_{3} \gamma_{4} \\
& -576 \gamma_{1}^{3} \gamma_{2} \gamma_{3} \gamma_{4} \\
& -221184 \lambda^{2} \gamma_{0}^{2} \gamma_{2}^{2} \gamma_{3} \gamma_{4} \\
& -55296 \lambda \gamma_{0} \gamma_{1} \gamma_{2}^{2} \gamma_{3} \gamma_{4}-3456 \gamma_{1}^{2} \gamma_{2}^{2} \gamma_{3} \gamma_{4} \\
& -73728 \lambda \gamma_{0} \gamma_{2}^{3} \gamma_{3} \gamma_{4} \\
& -9216 \gamma_{1} \gamma_{2}^{3} \gamma_{3} \gamma_{4}-9216 \gamma_{2}^{4} \gamma_{3} \gamma_{4} \\
& -294912 \lambda^{4} \gamma_{0}^{4} \gamma_{4}^{2}-147456 \lambda^{3} \gamma_{0}^{3} \gamma_{1} \gamma_{4}^{2} \\
& -27648 \lambda^{2} \gamma_{0}^{2} \gamma_{1}^{2} \gamma_{4}^{2} \\
& -2304 \lambda \gamma_{0} \gamma_{1}^{3} \gamma_{4}^{2}-80 \gamma_{1}^{4} \gamma_{4}^{2} \\
& -589824 \lambda^{3} \gamma_{0}^{3} \gamma_{2} \gamma_{4}^{2}-221184 \lambda^{2} \gamma_{0}^{2} \gamma_{1} \gamma_{2} \gamma_{4}^{2} \\
& -27648 \lambda \gamma_{0} \gamma_{1}^{2} \gamma_{2} \gamma_{4}^{2} \\
& -1152 \gamma_{1}^{3} \gamma_{2} \gamma_{4}^{2}-442368 \lambda^{2} \gamma_{0}^{2} \gamma_{2}^{2} \gamma_{4}^{2} \\
& -110592 \lambda \gamma_{0} \gamma_{1} \gamma_{2}^{2} \gamma_{4}^{2} \\
& -6912 \gamma_{1}^{2} \gamma_{2}^{2} \gamma_{4}^{2} \\
& -147456 \lambda \gamma_{0} \gamma_{2}^{3} \gamma_{4}^{2}-18432 \gamma_{1} \gamma_{2}^{3} \gamma_{4}^{2} \\
& -18432 \gamma_{2}^{4} \gamma_{4}^{2}+1536 \lambda^{3} \gamma_{0}^{3} \gamma_{1}^{3} \gamma_{5}+128 \lambda^{2} \gamma_{0}^{2} \gamma_{1}^{4} \gamma_{5} \\
& -40 \lambda \gamma_{0} \gamma_{1}^{5} \gamma_{5}-2 \gamma_{1}^{6} \gamma_{5} \\
& +768 \lambda^{2} \gamma_{0}^{2} \gamma_{1}^{3} \gamma_{2} \gamma_{5}-256 \lambda \gamma_{0} \gamma_{1}^{4} \gamma_{2} \gamma_{5}-28 \gamma_{1}^{5} \gamma_{2} \gamma_{5} \\
& -384 \lambda \gamma_{0} \gamma_{1}^{3} \gamma_{2}^{2} \gamma_{5} \\
& -128 \gamma_{1}^{4} \gamma_{2}^{2} \gamma_{5}-192 \gamma_{1}^{3} \gamma_{2}^{3} \gamma_{5} \\
& +768 \lambda^{2} \gamma_{0}^{2} \gamma_{1}^{2} \gamma_{3} \gamma_{5} \\
& +192 \lambda \gamma_{0} \gamma_{1}^{3} \gamma_{3} \gamma_{5}+8 \gamma_{1}^{4} \gamma_{3} \gamma_{5} \\
& +768 \lambda \gamma_{0} \gamma_{1}^{2} \gamma_{2} \gamma_{3} \gamma_{5}+96 \gamma_{1}^{3} \gamma_{2} \gamma_{3} \gamma_{5} \\
& +192 \gamma_{1}^{2} \gamma_{2}^{2} \gamma_{3} \gamma_{5}-16 \gamma_{1}^{4} \gamma_{4} \gamma_{5} \\
& +768 \lambda^{2} \gamma_{0}^{2} \gamma_{1}^{2} \gamma_{5}^{2}+192 \lambda \gamma_{0} \gamma_{1}^{3} \gamma_{5}^{2} \\
& +4 \gamma_{1}^{4} \gamma_{5}^{2}
\end{aligned}
$$




$$
\begin{aligned}
& +768 \lambda \gamma_{0} \gamma_{1}^{2} \gamma_{2} \gamma_{5}^{2}+96 \gamma_{1}^{3} \gamma_{2} \gamma_{5}^{2} \\
& +192 \gamma_{1}^{2} \gamma_{2}^{2} \gamma_{5}^{2}-256 \lambda^{2} \gamma_{0}^{2} \gamma_{1}^{4} \gamma_{6}-64 \lambda \gamma_{0} \gamma_{1}^{5} \gamma_{6} \\
& +4 \gamma_{1}^{6} \gamma_{6}-256 \lambda \gamma_{0} \gamma_{1}^{4} \gamma_{2} \gamma_{6} \\
& +32 \gamma_{1}^{5} \gamma_{2} \gamma_{6}+64 \gamma_{1}^{4} \gamma_{2}^{2} \gamma_{6} \\
& -16 \gamma_{1}^{4} \gamma_{3} \gamma_{6}-64 \gamma_{1}^{4} \gamma_{4} \gamma_{6} \\
& -64 \gamma_{1}^{4} \gamma_{5} \gamma_{6}-128 \gamma_{1}^{4} \gamma_{6}^{2}
\end{aligned}
$$

The parameters $\gamma_{0,1,2, \ldots, 6}$ in these expressions represent the ambiguity in the parametrization of quantum metric (5). Let us note that these bulky formulas, anyway, admit a completely invariant result when used in the potential (73), providing the very compact Eq. (74).

\section{Appendix B}

Here we derive the equation $\operatorname{DM}(35)$. All equations like $\operatorname{DM}(35)$ are numbered according to Ref. [11]. For comparison reasons, the notations in this section also follows [11]. The principal difference with the main text conventions is the opposite sign for the metric signature.

The starting expression is

$\Delta S_{m M}=-\frac{1}{32 \pi^{2}} \int d^{4} y \sqrt{-g} m_{\mu \nu \rho \sigma} \ln (-\square) M^{\rho \sigma \mu \nu}$,

where $\mathrm{DM}(15)$ gives

$$
\begin{aligned}
& M^{\rho \sigma \mu \nu}=\frac{M \kappa^{2}}{8} \int d \tau^{\prime} \delta^{4}\left(y-x\left(\tau^{\prime}\right)\right)\left(2 \dot{x}^{\rho} \dot{x}^{\sigma} \dot{x}^{\mu} \dot{x}^{\nu}+\dot{x}^{\rho} \dot{x}^{\sigma} g^{\mu \nu}\right), \\
& m_{\mu \nu \rho \sigma}=\frac{m \kappa^{2}}{8} \int d \tau \delta^{4}(y-z(\tau))\left(2 \dot{z}_{\rho} \dot{z}_{\sigma} \dot{z}_{\mu} \dot{z}_{\nu}+\dot{z}_{\mu} \dot{z}_{\nu} g_{\rho \sigma}\right) .
\end{aligned}
$$

Hereafter, $\dot{x}_{\mu}=\frac{d x_{\mu}}{d \tau^{\prime}}$ and $\dot{z}_{\mu}=\frac{d z_{\mu}}{d \tau}$. Before substituting (101) into (100), we take into account that the massive source $M$ is static and situated at the origin, such that $x^{0}=\tau$ and $x^{k} \equiv 0$. Furthermore,

$g^{\mu v} \dot{z}_{\mu} \dot{z}_{v}=-1$

Then, the contractions in (100) give

$$
\begin{aligned}
\Delta S_{m M}= & -\frac{m M \kappa^{4}}{64 \cdot 32 \pi^{2}} \int d y^{0} d^{3} \mathbf{y} \sqrt{-g(y)} \\
& \times \int d \tau \delta^{4}(y-z(\tau)) \\
& \times\left(2 \dot{z}_{\rho} \dot{z}_{\sigma} \dot{z}_{\mu} \dot{z}_{v}+\dot{z}_{\rho} \dot{z}_{\sigma} g_{\mu \nu}\right) \\
& \times \ln (-\square) \int d \tau^{\prime} \delta\left(y^{0}-\tau^{\prime}\right) \delta^{3}(\mathbf{y}) \\
& \times\left(2 \delta_{0}^{\rho} \delta_{0}^{\sigma} \delta_{0}^{\mu} \delta_{0}^{\nu}+\delta_{0}^{\rho} \delta_{0}^{\sigma} g^{\rho \sigma}\right)
\end{aligned}
$$

$$
\begin{aligned}
= & -\frac{m M \kappa^{4}}{64 \cdot 32 \pi^{2}} \int d y^{0} d^{3} \mathbf{y} \sqrt{-g(y)} \\
& \times \int d \tau \delta\left(y^{0}-z^{0}(\tau)\right) \delta^{3}(\mathbf{y}-\mathbf{z}(\tau)) \\
& \times\left(g_{00} g^{\rho \sigma} \dot{z}_{\rho} \dot{z}_{\sigma}+2 \dot{z}_{0} \dot{z}_{0} g^{\rho \sigma} \dot{z}_{\rho} \dot{z}_{\sigma}+\right. \\
& \left.\times 2 g_{00} \dot{z}_{0} \dot{z}_{0}+4 \dot{z}_{0} \dot{z}_{0} \dot{z}_{0} \dot{z}_{0}\right) \\
& \times \ln (-\square) \int d \tau^{\prime} \delta\left(y^{0}-\tau^{\prime}\right) \delta^{3}(\mathbf{y}) .
\end{aligned}
$$

Taking into account (102), the formula (103) boils down to

$$
\begin{aligned}
\Delta S_{m M}= & -\frac{m M \kappa^{4}}{64 \cdot 32 \pi^{2}} \int d y^{0} d^{3} \mathbf{y} \sqrt{-g(y)} \\
& \times \int d \tau \delta\left(y^{0}-z^{0}\right) \delta^{3}(\mathbf{y}-\mathbf{z}(\tau)) \\
& \times\left(-g_{00}-2 \dot{z}_{0} \dot{z}_{0}+2 g_{00} \dot{z}_{0} \dot{z}_{0}+4 \dot{z}_{0} \dot{z}_{0} \dot{z}_{0} \dot{z}_{0}\right) \\
& \times \ln (-\square) \delta^{3}(\mathbf{y})
\end{aligned}
$$

Taking the integral over $y^{0}$ and changing the order of integration, we get $y^{0} \rightarrow \tau$. Similarly, taking $d^{3} \mathbf{y}$ results in $\mathbf{y} \rightarrow \mathbf{z}(\tau)$. Assuming non relativistic $z^{\mu}$, i.e., $z^{0}=\tau$ and $\dot{z}_{k}=0$, we get

$$
\begin{aligned}
\Delta S_{m M}= & -\frac{m M \kappa^{4}}{64 \cdot 32 \pi^{2}} \int d \tau \\
& \times\left(-g_{00}-2 \dot{z}_{0} \dot{z}_{0}-2 \dot{z}_{0} \dot{z}_{0}+4 \dot{z}_{0} \dot{z}_{0} \dot{z}_{0} \dot{z}_{0}\right) \\
& \times \ln (-\square) \delta^{3}(\mathbf{z}(\tau)) \\
= & -\frac{m M \kappa^{4}}{512 \pi^{2}} \int d \tau \frac{1}{4} \ln (-\square) \delta^{3}(\mathbf{z}(\tau)),
\end{aligned}
$$

that is four time more than $\operatorname{DM}(35)$. Correcting the missing factor of 4 , the coefficient in DM(43) is $-1 / 6$ instead of $7 / 12$. This gives a Newtonian potential in the form (74).

\section{References}

1. Y. Iwasaki, Quantum theory of gravitation vs. classical theoryfourth-order potential. Prog. Theor. Phys. 46, 1587 (1971)

2. J.F. Donoghue, Leading quantum correction to the Newtonian potential. Phys. Rev. Lett. 72, 2996 (1994). arXiv:gr-qc/9310024

3. J.F. Donoghue, General relativity as an effective field theory: the leading quantum corrections. Phys. Rev. D 50, 3874 (1994). arXiv:gr-qc/9405057

4. I.B. Khriplovich, G.G. Kirilin, Quantum power correction to the Newton law. J. Exp. Theor. Phys. 95, 981 (2002). arXiv:gr-qc/0207118

5. N.E.J. Bjerrum-Bohr, J.F. Donoghue, B.R. Holstein, Quantum gravitational corrections to the nonrelativistic scattering potential of two masses. Phys. Rev. D 67, 084033 (2003). Erratum: [Phys. Rev. D 71, 069903 (2005)]. arXiv:hep-th/0211072

6. J.F. Donoghue, The effective field theory treatment of quantum gravity. AIP Conf. Proc. 1483, 73 (2012). arXiv:1209.3511

7. J.A. Helayël-Neto, A. Penna-Firme, I.L. Shapiro, Scalar QED hPlanck corrections to the Coulomb potential. JHEP 01, 009 (2000). arXiv:hep-th/9910080 
8. K.A. Kazakov, P.I. Pronin, Gauge dependence of effective gravitational field. Phys. Rev. D 62, 044043 (2000). arXiv:hep-th/9912129

9. T.S. Gribouk, K.A. Kazakov, P.I. Pronin, Gauge dependence of effective gravitational field. 2. Point-like measuring device. Phys. Rev. D 69, 024005 (2004). arXiv:hep-th/0306233

10. M.J. Duff, Quantum corrections to the schwarzschild solution. Phys. Rev. D 9, 1837 (1974)

11. D.A.R. Dalvit, F.D. Mazzitelli, Geodesics, gravitons and the gauge fixing problem. Phys. Rev. D 56, 7779 (1997). arXiv:hep-th/9708102

12. M.J. Duff, Inconsistency of quantum field theory in curved spacetime, in Oxford Conference on Quantum Gravity, pp. 81-105. OUP Report number: ICTP/79-80/38

13. I.L. Shapiro, Polemic notes on IR perturbative quantum gravity. Int. J. Mod. Phys. A 24, 1557 (2009). arXiv:0812.3521

14. M. Bordag, G.L. Klimchitskaya, U. Mohideen, V.M. Mostepanenko, Advances in the Casimir effect (Oxford University Press, New York, 2009)

15. S.K. Lamoreaux, Review on the book [13]. Phys. Today 63, 50 (2010)

16. J.Q. Quach, Gravitational Casimir effect. Phys. Rev. Lett. 114, 081104 (2015) [Erratum: Phys. Rev. Lett. 118, 139901 (2017)]. arXiv:1502.07429

17. T.G. Ribeiro, I.L. Shapiro, Scalar model of effective field theory in curved space. JHEP 1910, 163 (2019). arXiv:1908.01937

18. A.V. Manohar, Effective field theories. Lect. Notes Phys. 479, 311 (1997). arXiv:hep-ph/9606222

19. V. Ilisie, Concepts in quantum field theory. A practitioner's toolkit (Springer, Cham, 2016)

20. J.Z. Simon, Higher-derivative Lagrangians, nonlocality, problems, and solutions. Phys. Rev. D 41, 3720 (1990)

21. C.P. Burgess, Quantum gravity in everyday life: General relativity as an effective field theory. Living Rev. Relativ. 7, 5 (2004). arXiv:gr-qc/0311082

22. I.L. Buchbinder, I.L. Shapiro, Introduction to quantum field theory with applications to quantum gravity (Oxford University Press, Oxford, 2021)

23. J.D. Gonçalves, T. de Paula Netto, I.L. Shapiro, Gauge and parametrization ambiguity in quantum gravity. Phys. Rev. D 97, 026015 (2018). arXiv: 1712.03338

24. B.L. Giacchini, T. de Paula Netto, I.L. Shapiro, Vilkovisky unique effective action in quantum gravity. Phys. Rev. D 102, 106006 (2020). arXiv:2006.04217

25. G.B. Peixoto, A. Penna-Firme, I.L. Shapiro, One loop divergences of quantum gravity using conformal parametrization. Mod. Phys. Lett. A 15, 2335 (2000). arXiv:0103043

26. A.O. Barvinsky, G.A. Vilkovisky, The generalized SchwingerDeWitt technique in gauge theories and quantum gravity. Phys. Rep. 119, 1 (1985)

27. B.S. DeWitt, Dynamical theory of groups and fields (Gordon and Breach, New York, 1965)

28. G.A. Vilkovisky, The unique effective action in quantum field theory. Nucl. Phys. B 234, 125 (1984)

29. N. Burzillà, B.L. Giacchini, T. de Paula Netto, L. Modesto, Newtonian potential in higher-derivative quantum gravity. Phys. Rev. D 103, 064080 (2021). arXiv:2012.06254

30. N. Burzillà, B.L. Giacchini, T. de Paula Netto, L. Modesto, Higherorder regularity in local and nonlocal quantum gravity. Eur. Phys. J. C 81, 462 (2021). arXiv:2012.11829

31. L. Modesto, T. de Paula Netto, I.L. Shapiro, On Newtonian singularities in higher derivative gravity models. JHEP 04, 098 (2015). arXiv: 1412.0740

32. B.L. Giacchini, T. de Paula Netto, Weak-field limit and regular solutions in polynomial higher-derivative gravities. Eur. Phys. J. C 79, 217 (2019). arXiv:1806.05664
33. B.L. Giacchini, T. de Paula Netto, Effective delta sources and regularity in higher-derivative and ghost-free gravity. JCAP 07, 013 (2019). arXiv: 1809.05907

34. E.S. Fradkin, A.A. Tseytlin, Renormalizable asymptotically free quantum theory of gravity. Nucl. Phys. B 201, 469 (1982)

35. I.L. Shapiro, A.G. Jacksenaev, Gauge dependence in higher derivative quantum gravity and the conformal anomaly problem. Phys. Lett. B 324, 286 (1994)

36. B.L. Giacchini, T. de Paula Netto, I.L. Shapiro, On the VilkoviskyDeWitt approach and renormalization group in effective quantum gravity. JHEP 10, 011 (2020). arXiv:2009.0412

37. T. Taylor, G. Veneziano, Quantum gravity at large distances and the cosmological constant. Nucl. Phys. B 345, 210 (1990)

38. D.C. Rodrigues, P.S. Letelier, I.L. Shapiro, Galaxy rotation curves from general relativity with quantum corrections. JCAP 04, 020 (2010). arXiv:0911.4967

39. A. Babic, B. Guberina, R. Horvat, H. Stefancic, Renormalizationgroup running cosmologies. A scale-setting procedure. Phys. Rev. D 71, 124041 (2005). arXiv:astro-ph/0407572

40. S. Domazet, H. Stefancic, Renormalization group scale-setting in astrophysical systems. Phys. Lett. B 703, 1 (2011). arXiv: 1010.3585

41. J.M. Cline, S. Jeon, G.D. Moore, The phantom menaced: constraints on low-energy effective ghosts. Phys. Rev. D 70, 043543 (2004). arXiv:hep-ph/0311312

42. T.D. Lee, G.C. Wick, Negative metric and the unitarity of the $S$ matrix. Nucl. Phys. B 9, 209 (1969)

43. T.D. Lee, G.C. Wick, Finite theory of quantum electrodynamics. Phys. Rev. D 2, 1033 (1970)

44. L. Modesto, I.L. Shapiro, Superrenormalizable quantum gravity with complex ghosts. Phys. Lett. B 755, 279 (2016). arXiv: 1512.07600

45. L. Modesto, Super-renormalizable or finite Lee-Wick quantum gravity. Nucl. Phys. B 909, 584 (2016). arXiv:1602.02421

46. D. Anselmi, On the quantum field theory of the gravitational interactions. JHEP 06, 086 (2017). arXiv: 1704.07728

47. M. Asorey, J.L. Lopez, I.L. Shapiro, Some remarks on high derivative quantum gravity. Int. J. Mod. Phys. A 12, 5711 (1997). arXiv:hep-th/9610006

48. D. Anselmi, M. Piva, A new formulation of Lee-Wick quantum field theory. JHEP 06, 066 (2017). arXiv:1703.04584

49. D. Anselmi, M. Piva, Perturbative unitarity of Lee-Wick quantum field theory. Phys. Rev. D 96, 045009 (2017). arXiv:1703.05563

50. D. Anselmi, Fakeons and Lee-Wick models. JHEP 02, 141 (2018). arXiv: 1801.00915

51. Y.V. Kuz'min, The convergent nonlocal gravitation (in Russian). Sov. J. Nucl. Phys. 50, 1011 (1989)

52. Y.V. Kuz'min, Yad. Fiz. 50, 1630 (1989)

53. L. Modesto, Super-renormalizable Quantum Gravity. Phys. Rev. D 86, 044005 (2012). arXiv: 1107.2403

54. L. Modesto, L. Rachwal, Super-renormalizable and finite gravitational theories. Nucl. Phys. B 889, 228 (2014). arXiv:1407.8036

55. L. Modesto, L. Rachwal, Nonlocal quantum gravity: a review. Int. J. Mod. Phys. D 26, 1730020 (2017)

56. F. Briscese, L. Modesto, Cutkosky rules and perturbative unitarity in Euclidean nonlocal quantum field theories. Phys. Rev. D 99, 104043 (2019). arXiv:1803.08827

57. F. Briscese, L. Modesto, Non-unitarity of Minkowskian nonlocal quantum field theories. Eur. Phys. J. C 81, 730 (2021). arXiv:2103.00353

58. F. Briscese, L. Modesto, Unattainability of the trans-Planckian regime in nonlocal quantum gravity. JHEP 09, 056 (2020). arXiv:1912.01878 
59. L. Rachwał, L. Modesto, A. Pinzul, I.L. Shapiro, Renormalization group in six-derivative quantum gravity. arXiv:2104.13980

60. F. Briscese, L. Modesto, Nonlinear stability of Minkowski spacetime in Nonlocal Gravity. JCAP 07, 009 (2019). arXiv:1811.05117

61. F. Briscese, G. Calcagni, L. Modesto, Nonlinear stability in nonlocal gravity. Phys. Rev. D 99, 084041 (2019). arXiv:1901.03267

62. L. Modesto, The Higgs mechanism in nonlocal field theory. JHEP 06, 049 (2021). arXiv:2103.05536

63. L. Modesto, Nonlocal spacetime-matter. arXiv:2103.04936

64. A. Smailagic, E. Spallucci, Lorentz invariance, unitarity in UV-finite of QFT on noncommutative spacetime. J. Phys. A 37, 1 (2004). [Erratum: J. Phys. A 37, 7169 (2004)]. arXiv:hep-th/0406174
65. J. Liu, Q. Li, F. Briscese, G. Calcagni, L. Modesto, work in progress

66. K.S. Stelle, Renormalization of higher derivative quantum gravity. Phys. Rev. D 16, 953 (1977)

67. Wolfram's Mathematica, Version 12.0 (Wolfram Research, Champaign, 2019)

68. J.M. Martín-García, xAct: efficient tensor computer algebra for the Wolfram Language. http://xact.es

69. T. Nutma, xTras: a field-theory inspired xAct package for mathematica. Comput. Phys. Commun. 185, 1719 (2014). arXiv: 1308.3493 